\title{
Knockdown of Fidgetin Improves Regeneration of Injured Axons by a Microtubule-Based Mechanism
}

\author{
๑DAndrew J. Matamoros, ${ }^{1}$ ○Veronica J. Tom, ${ }^{1}$ Di Wu, ${ }^{1}$ Yash Rao, ${ }^{1}$ David J. Sharp, ${ }^{2 *}$ and $\odot$ Peter W. Baas ${ }^{1 *}$ \\ ${ }^{1}$ Department of Neurobiology and Anatomy, Drexel University College of Medicine, Philadelphia, Pennsylvania 19129, and ${ }^{2}$ Albert Einstein College of \\ Medicine, Department of Physiology and Biophysics, Bronx, New York 10461
}

Fidgetin is a microtubule-severing protein that pares back the labile domains of microtubules in the axon. Experimental depletion of fidgetin results in elongation of the labile domains of microtubules and faster axonal growth. To test whether fidgetin knockdown assists axonal regeneration, we plated dissociated adult rat DRGs transduced using AAV5-shRNA-fidgetin on a laminin substrate with spots of aggrecan, a growth-inhibitory chondroitin sulfate proteoglycan. This cell culture assay mimics the glial scar formed after CNS injury. Aggrecan is more concentrated at the edge of the spot, such that axons growing from within the spot toward the edge encounter a concentration gradient that causes growth cones to become dystrophic and axons to retract or curve back on themselves. Fidgetin knockdown resulted in faster-growing axons on both laminin and aggrecan and enhanced crossing of axons from laminin onto aggrecan. Strikingly, axons from within the spot grew more avidly against the inhibitory aggrecan concentration gradient to cross onto laminin, without retracting or curving back. We also tested whether depleting fidgetin improves axonal regeneration in vivo after a dorsal root crush in adult female rats. Whereas control DRG neurons failed to extend axons across the dorsal root entry zone after injury, DRG neurons in which fidgetin was knocked down displayed enhanced regeneration of axons across the dorsal root entry zone into the spinal cord. Collectively, these results establish fidgetin as a novel therapeutic target to augment nerve regeneration and provide a workflow template by which microtubule-related targets can be compared in the future.

Key words: axon; DRG; fidgetin; microtubule; regeneration

Significance Statement

Here we establish a workflow template from cell culture to animals in which microtubule-based treatments can be tested and compared with one another for their effectiveness in augmenting regeneration of injured axons relevant to spinal cord injury. The present work uses a viral transduction approach to knock down fidgetin from rat neurons, which coaxes nerve regeneration by elevating microtubule mass in their axons. Unlike previous strategies using microtubule-stabilizing drugs, fidgetin knockdown adds microtubule mass that is labile (rather than stable), thereby better recapitulating the growth status of a developing axon.

\section{Introduction}

Regeneration of injured adult axons is severely limited in the CNS. An injured axon in the adult CNS tends to retract and

Received July 24, 2018; revised Nov. 21, 2018; accepted Dec. 24, 2018.

Author contributions: A.J.M. wrote the first draft of the paper; A.J.M., V.J.T., and P.W.B. edited the paper; A.J.M., V.J.T., D.W., D.J.S., and P.W.B. designed research; A.J.M. performed research; A.J.M. contributed unpublished reagents/analytic tools; A.J.M. and Y.R. analyzed data; A.J.M. and P.W.B. wrote the paper.

This work was supported by Craig H. Neilsen Foundation Grant 259350 to P.W.B., Telemedicine and Advanced Technology Research Center at the U.S. Army Medical Research and Materiel Command Award W81XWH1210379 to D.J.S., National Institutes of Health, National Institute of Neurological Disorders and Stroke Grant R01 NS28785 to P.W.B., Grant R01 GM109909 to D.J.S., and Grants R01 NS085426 and R01 NS106908 to V.J.T. A.J.M. was supported by National Institutes of Health, National Institute of Neurological Disorders National Research Service Award Predoctoral Fellowship 1 F31 NS103443-01A1.

D.J.S. is Chief Scientific Officer and P.W.B. is a member of the Scientific Board of Microcures, a biotechnology company that has identified fidgetin as a potential target for therapeutic applications. The remaining authors declare no competing financial interests.

${ }^{*}$ D.J.S. and P.W.B. contributed equally to this work as co-senior authors.

Correspondence should be addressed to Peter W. Baas at pwb22@drexel.edu. degenerate, rather than regenerate, both because the intrinsic growth potential of the adult axon is reduced relative to a juvenile axon and because growth is impeded by chemical and physical obstacles, such as scar tissue and injury-associated inhibitory molecules (Tran et al., 2018). Also impeding their growth, regenerating axons tend to form inappropriate synapses with glial cells within the dorsal root entry zone (DREZ) (Di Maio et al., 2011). An intervention is needed to enable axons to overcome these intrinsic and extrinsic barriers and grow toward appropriate targets.

Microtubules have become a promising target for therapy, especially given that recent studies show that treatment with microtubule-stabilizing drugs improves axonal regeneration in rats with injured spinal cords (Hellal et al., 2011; Ruschel et al., 
2015; Ruschel and Bradke, 2018; Sandner et al., 2018). As for any direct effects on the axon, the drugs may positively affect regeneration because stabilization of microtubules prevents axonal retraction and because stabilized microtubules at their polymerizing ends may enable the axon's tip to push through normally inhibitory environments. However, these effects do not reflect how axonal growth normally occurs or how the tip of the axon, termed the growth cone, normally functions. Indeed, other studies suggest that the key to axonal regeneration may be the opposite of stabilization of microtubules, which is to shift a predominantly stable microtubule array into a more dynamic one (Bradke et al., 2012). In support of this, axons regenerate better when they recapitulate characteristics of labile microtubules, such as being less acetylated and detyrosinated (Cho and Cavalli, 2012; Gobrecht et al., 2016).

Individual microtubules in the axon consist of a stable domain and a labile domain, the latter of which is notably more dynamic and less acetylated than the former (Baas and Black, 1990; Baas et al., 1991, 2016). During development, a palette of microtubulerelated proteins contributes to regulating axonal growth, with some of these proteins regulating the levels and proportions of the microtubule array that is stable or labile. We have recently proposed that a potentially powerful approach for augmenting regeneration of injured CNS axons would be to add labile microtubule mass (Matamoros and Baas, 2016). Depleting or inhibiting proteins that normally suppress the elongation of the labile domains of microtubules could theoretically accomplish this. One such protein is fidgetin, a microtubule-severing protein that, by favoring less acetylated regions of microtubules, preferentially cuts the microtubule in its labile domain (Leo et al., 2015; Austin et al., 2017). Depleting fidgetin from fetal neurons results in faster-growing axons with a higher content of microtubules, a greater fraction of which is labile and less acetylated. Similar results were observed when fidgetin was depleted from adult rat DRG neurons, as well as enhanced growth of axons onto stripes of aggrecan, one of the inhibitory proteins that comprises the scar tissue of the injured spinal cord (Austin et al., 2017).

Here our primary goal was to expand our testing of fidgetin as a novel microtubule-based target to augment regeneration of injured axons in the CNS. A secondary goal was to create a workflow template that can be used in the future to test other microtubule-related targets in a consistent manner so that the effects can be compared with one another. For this, we started with a significant modification of a previously developed cell culture assay for testing the capacity of adult DRG axons to overcome the inhibitory molecules related to the injured milieu. Our version of the assay enables acquisition and analysis of a far greater volume of data, which is important given the heterogeneity in these cultures. From there, we used an in vivo assay on rats which involves a crush injury to the central branch of the axons of the DRG.

\section{Materials and Methods}

AAV5-based shRNA constructs. For knockdown of fidgetin, a construct termed AAV5-U6-shFIGN-CMV-GFP (referred to hereafter as shFIGN), was custom-made by Vector Biolabs and provided to us together with a control construct termed AAV5-U6-controlRNA-CMV-GFP (referred to hereafter as Control). The cell-based shRNA validation service of Vector Biolabs was used to validate the shRNA. For this, the cDNA of the gene of interest, rat fidgetin (NM_001106484), was cloned into a validation vector. A total of six predicted shRNA sequences were cloned individually into different shRNA vectors. Packaging cells were transfected with both the rat fidgetin cDNA and shRNA vectors, and gene knockdown was assessed using qRT-PCR from cellular lysate of the cotransfected cells. Of the six sequences tested, clone \#6 (CACC GCAA GATTCAGCCTAGCATATC TCGA GATATGCTAGGCTGAATCT TGC TTTT; targeting sequence underlined) had the greatest knockdown efficiency (96\%) and, hence, was selected for viral production. The $0.4 \mathrm{~kb}$ U6-shRNA expression cassette was inserted between BamHI/NotI sites on the pAAV-GFP-BASIC plasmid sold by Vector Biolabs (plasmid name: pAAV-GFP-U6-r-FIGN-shRNA). CMV promoter drove expression of the GFP reporter gene.

Vector Biolabs uses the Ad-HQ system to produce their adenoassociated virus (AAV). Our gene of interest was cloned into a shuttle vector and confirmed using restriction digestion. The expression cassette was transferred to the adenovirus vector and was confirmed using both restriction mapping and sequence verification. The recombinant adenoviral DNA is transfected into packaging cells and virus is subsequently amplified, titrated, and in vivo preps are subjected to $\mathrm{CsCl}$ purification.

Assessment of fidgetin knockdown. Rat fibroblasts (RFL-6) were used to assess the ability of the shRNA plasmid to knockdown ectopically expressed fidgetin-GFP. The mEmerald-fidgetin (termed FIGN-GFP; mEmerald is a modified GFP), generated from rat fidgetin cDNA (NM_001106484), was provided by W. Frankel. The pAAV-GFP-U6-rFIGN-shRNA or pAAV-GFP-U6-Scramble-shRNA and FIGN-GFP expression vectors were conucleofected into cells using the Lonza Nucleofector. Cells were cultured for 24 and $48 \mathrm{~h}$. Cell lysates were collected using RIPA lysis solutions from Santa Cruz Biotechnology and a Pierce BCA protein kit to measure protein concentrations of samples. The Bio-Rad Electrophoresis Vertical Apparatus and Mini-PROTEAN precast gels were used for SDS-PAGE, and the Bio-Rad Trans-Blot Turbo Transfer System was used to transfer proteins to a nitrocellulose membrane for Western blotting. A protein volume of $10 \mu \mathrm{g}$ was loaded into the precast gels. The Pierce ECL Western Blotting Substrate kit was used to image membranes on both traditional cl-xposure film and direct detection via the Bio-Rad Chemiluminescence Imager (ChemiDoc). Blots were stained for fidgetin and GAPDH. GAPDH was used as the loading control to compare evenly loaded protein samples. The polyclonal rabbit anti-fidgetin (Santa Cruz Biotechnology; SC-68343) and monoclonal mouse anti-GAPDH (ab8245) primary antibodies were used. Secondary HRP-conjugated antibodies from Jackson ImmunoResearch Laboratories were used to detect the host species of the primary antibodies (antimouse 115-035-146 and anti-rabbit 111-035-144).

To confirm that the shRNA can knock down fidgetin from adult DRG neurons, we used a lentivirus containing mCherry-Fidgetin (NM_001106484), provided by Dr. Shen Lin of Temple University. The primary cultures were grown as described below, with the exception that the neurons were cultured with both lentivirus containing mCherryFIGN and AAV5-shFIGN or AAV5-Control. Cells were exposed to both lentivirus and AAV5 after $1 \mathrm{~d}$ of primary culture plating. After $3 \mathrm{~d}$, neurons began to strongly express both AAV5-GFP reporter and mCherryFIGN. Cells were imaged using a Carl Zeiss Z1.Observer fitted with a live-cell incubation system complete with $\mathrm{CO}_{2}$ and climate control. A temperature of $32^{\circ} \mathrm{C}$ was maintained during imaging. Images were acquired using the Carl Zeiss mRm Axiocam and Carl Zeiss Zen Blue software. High-quality images were exported and analyzed in ImageJ for mCherry fluorescence integrated density to assess the knockdown efficiency of our AAV5 shFIGN to deplete ectopically expressed mCherry-FIGN.

Modified aggrecan spot assay for studies on cultured adult rat DRGs. A cell culture assay, modified from a previously developed assay (Wu et al., 2016), was used to measure axonal regeneration across an inhibitory gradient in vitro. Adult male and female rats (6-7 months) were killed using $\mathrm{CO}_{2}$, and spinal cords were dissected immediately thereafter. A total of 50 DRGs from C1 to L5 were dissected from the spinal cord on ice using a dissecting microscope, fine forceps, and micro-scissors. DRGs were kept in dissecting medium on ice and then treated with chondroitinase (0.25\%; Worthington) and trypsin (0.25\%; Worthington). Enzymatically digested DRGs were washed with culture medium containing $10 \%$ FBS and then gently triturated into a single-cell suspension using a fire-polished glass pipette, after which the dispersed cell suspension was filtered through mesh cloth to remove cellular debris before being seeded onto plastic culture dishes. The addition of B27 and NGF over FBS 
(which improved neuronal survival), as well as the use of a mesh filter to clear myelin debris (the presence of which makes cultures less healthy and obstructs visualization of cells in the microscope) were based on previously published work (Gundersen and Barrett, 1980; Delree et al., 1989; Tom et al., 2004; Wu et al., 2017).

The cells were counted using a hemocytometer; and across all experiments, an average of 150,000 neurons were obtained from a single rat's dissection of 48 DRG from C2 to L5. Cells were scored as neurons on the basis of their much larger size compared with other cell types. No effort was made to remove satellite glia or other non-neuronal cells. Cells were evenly distributed across two poly-D-lysine $(0.1 \mathrm{mg} / \mathrm{ml})$ and laminin $(5$ $\mu \mathrm{g} / \mathrm{ml}$ ) coated plastic-bottomed dishes for viral transduction; control and shFIGN AAV5. To avoid viral toxicity that can occur if cultures are immediately exposed to the virus, viral constructs were added to the cultures $24 \mathrm{~h}$ after plating. Cultures were incubated at $35^{\circ} \mathrm{C}$ for $8 \mathrm{~d}$ (the point at which GFP expression is highest in the neurons) in adult neuron culture medium supplemented with NGF, and then treated with Accutase for $30 \mathrm{~min}$ at $35^{\circ} \mathrm{C}$. The resulting loose cell suspension was further dissociated with gentle pipette trituration. All cells were removed from the surface of the dish, and the dish was rinsed three times with serumcontaining medium that was pooled together to assure all cells were recovered. Cells were seeded onto $20 \mathrm{~mm}$ glass-bottomed dishes with 7 spots of aggrecan dried on the poly-D-lysine $(0.1 \mathrm{mg} / \mathrm{ml})$ and laminin $(5 \mu \mathrm{g} / \mathrm{ml})$ coated glass surfaces.

The day before replating cells, Matek glass-bottomed dishes were coated with poly-D-lysine $(0.1 \mathrm{mg} / \mathrm{ml})$ for $24 \mathrm{~h}$ and rinsed with distilled water. An aggrecan (Sigma Aldrich; A1960) and laminin stock solution of $0.7 \mathrm{mg} / \mathrm{ml}$ aggrecan and $5 \mu \mathrm{g} / \mathrm{ml}$ laminin was made. On the PDL-coated glass surfaces, $2 \mu \mathrm{l}$ of the aggrecan and laminin stock solution was pipetted seven times, following a printed pattern with even spacing placed below the transparent glass bottom (see Fig. 1A). The dishes were left in the hood with their tops ajar to allow for evaporation. The spotted dishes were again coated with $5 \mu \mathrm{g} / \mathrm{ml}$ of laminin the day of replating. Dishes were rinsed before replating with warm culture medium. Before replating cells, counts were performed using a hemocytometer, and only neurons were counted. Cells were plated at a relatively low density of 100 neurons/dish to trace individual axons and not overwhelm the dish with neurite growth that was untraceable. The primary cultures are plated on the aggrecan spotted surface, and cells are agitated every $5 \mathrm{~min}$ for $30 \mathrm{~min}$ to distribute cells evenly across the glass surface. The dried spots of aggrecan and laminin, referred to as aggrecan spots, have an increasing concentration of aggrecan from the inside toward the outside, relative to the center of the spot. This is due to the "coffee ring" effects of drying the spots on the glass surface. The highest concentration of aggrecan is located at the edge of the spot, as can be visualized using fluorescence resonance heat mapping (see Fig. $1 B$ ).

Three days after replating onto aggrecan-spotted glass-bottomed dishes, cultures were rinsed with warm PBS. The PBS was aspirated to remove cellular debris and replaced with a $1 \times$ PHEM fixation solution containing 4\% PFA, 0.8\% glutaraldehyde (for improved microtubule fixation), and $0.1 \%$ Triton X-100. After $15 \mathrm{~min}$, cultures were rinsed with PBS to remove the fixation solution. To quench autofluorescence from the aldehydes used for fixation, ice-cold $2 \mathrm{mg} / \mathrm{ml}$ sodium borohydride solution was added to the fixed cells on ice for $15 \mathrm{~min}$, repeated, and rinsed with PBS. The fixed cells were then blocked with $10 \%$ normal goat serum, $0.1 \%$ BSA, and $0.1 \%$ Triton X-100 in PBS for $1 \mathrm{~h}$ at room temperature. The primary antibodies were incubated overnight at $4^{\circ} \mathrm{C}$; and for indirect immunofluorescence, secondary antibodies were applied for $2 \mathrm{~h}$ at room temperature. The cells were again rinsed, and wet mounting medium was applied. A 1.5 coverslip was placed over the mounting medium in the glass-bottomed dish and sealed with laboratory-grade nail polish.

A Carl Zeiss Z1.Observer equipped with a motorized stage and Carl Zeiss Axiocam MRm camera was used for fluorescence imaging of in vitro samples. Images were acquired at $10 \times$ for mosaic tiling. Carl Zeiss Zen Blue software and the motorized stage were used to tile a mosaic picture of the entire $20 \mathrm{~mm}$ glass-bottomed surface including four separate channels for Cy3 ( $\beta 3$-tubulin or total $\beta$-tubulin), AlexaFluor-488 (GFP), Cy5 (Aggrecan), and UV (DAPI). Images were loaded into ImageJ anal- ysis software, and the plugin NeuronJ (Image Biosciences) was used to measure axon length. To measure the total cell coverage (both neurons and satellite glia) of the spots, the tiled-mosaic images of entire glass surfaces of the culture dishes were opened in ImageJ and used to trace the individual aggrecan spots ( $n=7$ per dish) with ROIs using the Cy5 channel's image, which were saved in the ImageJ ROI manager. These ROIs were then overlaid on tiled-mosaic images of the Cy3 channel, so as to measure the amount of total $\beta$-tubulin fluorescence area coverage per aggrecan spot using ImageJ thresholding and area analysis. GFP ${ }^{+}$neurons are identified by their distinct morphology and $\beta 3$-tubulin costaining, which allowed us to count the number of neurons per spot as well as the number of axons crossing the aggrecan spot's border. The total number of non-neuronal cells were counted using DAPI staining. Cell counts off the spot used the same ROIs of the aggrecan spots ( $n=7$ per dish), except in this case they were shifted adjacent to the aggrecan spot, so as to measure cells off the spot. Cell counts were performed using ImageJ.

Direct and indirect antibody techniques were used for immunocytochemistry. For axonal length analysis, a polyclonal rabbit anti- $\beta$ III tubulin IgG antibody conjugated to Cy3 (Millipore; AB15708C3) was used to label neuronal microtubules. When analyzing both neurons and satellite glia, a monoclonal mouse anti- $\beta$ tubulin IgG antibody conjugated to Cy3 (ab11309: Abcam) was used to label total $\beta$ tubulin ( $\beta 1-5$ subunits). To detect the AAV5 reporter GFP protein, a polyclonal anti-GFP IgG antibody conjugated to AlexaFluor-488 (Thermo Fisher Scientific; A-21311) was used. The spots of aggrecan were visualized using the mouse monoclonal anti-chondroitin sulfate proteoglycan IgM antibody (Sigma Aldrich; C8035) and a secondary goat anti-mouse IgM antibody conjugated to Cy5 (Jackson ImmunoResearch Laboratories; 115-175-075). The use of both IgG and IgM antibodies allowed for triple immunolabeling. The nuclear and chromosome counterstain DAPI (Thermo Fisher Scientific; D1306) was used to count cells (Thermo Fisher Scientific; D1306) in combination with the above-mentioned antibodies. Antibodies were combined for double and triple immunolabeling of cell cultures.

In vivo dorsal root crush assay. The dorsal root crush assay was conducted similarly to previously published studies (Wu et al., 2016; Cheah et al., 2017) on adult female Sprague Dawley rats (Taconic Farms). Rats were anesthetized with isoflurane using an isoflurane pump. Once the animals were unconscious, the skin above the cervical vertebrae was shaved and sterilized with $\beta$ iodine solution. Optical ointment was applied to the eyes. A nose cone was fixed to the animal's head with a steady flow of isoflurane mixed with oxygen being administered via the pump. A small incision was made into the neck of the rat using a surgical scalpel. Muscle was gently removed with forceps and tissue separators. Care was taken to not damage the surrounding tissue. Saline solution was regularly applied to the separated muscle, and just enough room was made to insert rongeurs, forceps, and the glass micropipette for conducting the laminectomy, crush, and DRG injections, respectively. The cervical spine was exposed, and a laminectomy was performed from $\mathrm{T} 1$ to $\mathrm{C} 5$, exposing the corresponding dorsal spinal cord and dorsal roots. Enough of the bone was removed to also reveal the right C6, C7, and C8 DRGs for AAV microinjection. The dorsal roots of the right DRG from C5 to T1 were crushed with fine \#5 Dumont forceps (11251-35; Fine Science Tools). The meninges were punctured using a small needle, and one of the sides of the Dumont forceps was carefully placed under the dorsal root, with care not to shake, pull, or damage any surrounding tissue and blood vessels. The crush was held for $10 \mathrm{~s}$, lifted, and crushed again for $10 \mathrm{~s}$. Dorsal roots were crushed, not severed. The DRGs were injected with AAV5 using a beveled-tip glass micropipette fixed to the needle of a Hamilton syringe using caulk. AAV5 was microinjected at a rate of 0.1 $\mu \mathrm{l} / \mathrm{min}$ and a total volume of $1 \mu \mathrm{l} / \mathrm{DRG}$ for the right C6, C7, and C8 DRGs.

Absorbent micro-pads were placed over the exposed cord, and muscles overlaying the cord were sutured. The skin was stapled shut with surgical staples (12020-00; Fine Science Tools). Animals were given an injection of slow-release buprinex for pain and ampicillin as the antibiotic. Animals were kept on a heating pad overnight with only one-third of the cage on the pad. For the sham surgeries, all the same surgical steps were followed, except for the crush. Injured and sham-surgery animals were provided with gelatin-food and water, ad libitum. The following 
day, animals were given their normal chow, and the cages were exchanged. Animals were inspected for loss of sensation in the front-right forelimb by observing characteristic curling of the front-right paw and arm inwards, toward the rat's chest. Animals were inspected daily, including the 30th day when animals were killed. As a first pass toward assessing the potential for functional recovery, qualitative observations were made on whether or not the animal was bearing weight on the injured paw.

After $30 \mathrm{~d}$, the animals were killed with Euthasol and immediately placed in the chemical fume hood for saline and PFA perfusions. A \#gauge needle was inserted at the bottom of the still-beating heart and slid into the aorta. A $250 \mathrm{ml}$ saline solution was perfused through the animal to clear the spinal cord of blood. Subsequently, a $250 \mathrm{ml}$ 4\% PFA solution was perfused through the animal for tissue fixation. The spinal cords and DRGs were removed under a dissecting microscope and placed in $4 \%$ PFA solution overnight at $4^{\circ} \mathrm{C}$. The following day, the fixative was replaced with $30 \%$ sucrose and again stored at $4^{\circ} \mathrm{C}$. After $48 \mathrm{~h}$, the sucrose solution was replaced with fresh sucrose solution, and the tissue was stored for at least 1 week at $4^{\circ} \mathrm{C}$ to assure adequate sucrose absorption by the tissue.

The spinal cords and DRGs were flash frozen using 2-methyl butane on dry ice. The sections were first placed in a Tissue Tek container, and Tissue Tek OCT compound (50-363-579; Thermo Fisher Scientific) was used to cover the tissue. After flash freezing at $-50^{\circ} \mathrm{C}$, tissue was stored in a $-80^{\circ} \mathrm{C}$ freezer until tissue sectioning. Spinal cord blocks were placed in the cryostat to be used the night before sectioning, to allow tissue to adjust to the same temperature as the cryostat. Sections of the dorsal spinal cord (preferably with dorsal roots still attached) were cut at a thickness of $20 \mu \mathrm{m}$ and the DRG at $15 \mu \mathrm{m}$. Sections were placed in cryoprotectant solution containing thimersol and stored at $4^{\circ} \mathrm{C}$ until immunohistochemistry.

For immunohistochemistry, we used the monoclonal mouse antiNeuN (Millipore; MAB377), polyclonal rabbit anti-p75 (Abcam; ab8874), and polyclonal rabbit anti-GFP (Abcam; ab6556) primary antibodies. The anti-p75 antibody was used to visualize NGF receptors on peripheral satellite glia to demark the DREZ, where the peripheral nervous system (dorsal root) meets the central nervous system (dorsal horn). The anti-NeuN antibody was used along with the anti-GFP antibody to label GFP ${ }^{+}$neurons in the DRGs. The anti-GFP antibody was also used to label the $\mathrm{GFP}^{+}$axons attempting to regenerate across the DREZ and back into the dorsal horn.

Immunolabeled DRG sections were imaged using a Carl Zeiss Z1.Observer epifluorescence microscope, and a Carl Zeiss Axiocam $\mathrm{MRm}$ camera was used. Images were acquired at $20 \times$ for mosaic tiling. Carl Zeiss Zen Blue software was used to quantify the number of $\mathrm{NeuN}^{+}$ and $\mathrm{GFP}^{+}$cells to calculate percentage transduction. Immunolabeled spinal cord sections were imaged using a Leica SP8 Confocal Microscope and LAS X software. Tiled $z$-stack images were obtained, and maximum intensity projection images were output to ImageJ. NeuronJ (Image Biosciences) was used to trace the $\mathrm{GFP}^{+}$axons that extended beyond the DREZ, demarcated by $\mathrm{p} 75^{+}$Schwann cells.

RNA Scope (ACDbio) was used to image fidgetin mRNA, according to the manufacturer's instructions. Tissue sections were dehydrated and exposed to probes targeting fidgetin and PPIB (positive control). Subsequent amplification steps were used to amplify the fluorescent dye to allow for single mRNA imaging. Fluorescent puncta represent individual mRNA, whereas fluorescent aggregates are comprised of multiple mRNAs.

Experimental design, statistical analysis, and reporting. All data were recorded and saved in Microsoft Excel. Data collection was validated using blinded, inter-rater reliability testing. An aim of this investigation was to develop a medium-throughput regimen for target screening that included both in vitro and in vivo assays. Therefore, for our in vitro experiments, we included sample sizes that were sufficient to detect differences based on Power analysis, pilot data, and reproducibility. The sample size for the in vivo experiments were based on similar rodent regeneration studies, and responsible animal usage was considered. All samples sizes are reported in Results. Statistical analyses were performed using SPSS Statistics 24 (IBM) where the distribution of each dataset was tested for normality (Kolmogorov-Smirnov and Shapiro-Wilks Normality Testing), and histograms were compared across groups. Outliers were removed if they were extreme $(>3 \times$ the SD from the mean; flagged by SPSS, IBM). All data were approximately normally distributed, and variances were approximately equal according to Levene's test of Homogeneity of Variance and visual observations, unless stated otherwise in Results. Data that were not normally distributed underwent nonparametric analysis. All data are presented as mean \pm SEM, even when medians were compared. Statistical testing included the $t$ test and the nonparametric Mann-Whitney $U$ test was conducted when normality was violated. The Welch $t$ test was used when homogeneity of variance was violated. Data that violated normality did so because of the nature of the heterogeneity of primary adult cultures. Histological analysis of axon crossing found that homogeneity of variance was violated most likely due to the large amount of 0 values for the control group. All $p$ values are reported with their appropriate test statistics in Results.

\section{Results}

\section{Modified aggrecan spot assay for adult DRG neurons}

Primary cultures of adult rodent DRGs have been used for decades as the preferred in vitro model to assess axonal regeneration (Tom et al., 2004; Podratz et al., 2011; Lin et al., 2015; Wu et al., 2016; Wang et al., 2017), in part because they are one of the few types of adult nerve tissue that is amenable to cell culture. Even so, they are problematic because each DRG culture contains few neurons relative to satellite cells and other non-neuronal cells and because there are three distinct types of neurons with very different characteristics in each DRG. In a typical dissociated culture, some neurons are bipolar, others are monopolar, and still others are bushy in morphology. In some past studies, DRGs have been cultured as explants, such that the morphology of individual neurons is concealed in the explant, with axons emanating from the explant as a dense halo. A difficulty with explant cultures is that axonal growth from the explants can vary, depending on how much of the capsule surrounding the ganglion is removed and how well the explant is adhered to the substrate.

In terms of challenging the axons to regenerate in an injurylike environment, molecules of chondroitin sulfate proteoglycans (CSPGs), such as aggrecan, have been dried to the substrate as strips or as spots, with a strip providing a sharp demarcation between laminin and CSPG and spots providing a concentration gradient (for the small number of neurons that adhere on the spot as opposed to off the spot) that is more reminiscent of the challenge facing the injured axon in vivo (Tom et al., 2004; Lin et al., 2015; Wu et al., 2016). We sought to modify the spot assay to produce a greater amount of data than previously obtained with these cultures, so that the analyses and statistics would be robust despite the inherent variability. For this, we placed 7 spots of aggrecan in each culture dish and used a contemporary tiling function on our imaging system to image the entire culture on the 20-mm-diameter glass surface for subsequent quantification of every neuron, both on and off the aggrecan spots. The assay enables us to investigate various parameters, such as the number of neurons extending axons, axons crossing the inhibitory border, the length of axons both on and off the spots, and the reaction of axons to the gradient of aggrecan; and to gather large amounts of data often required to achieve statistical significance in light of the natural heterogeneity of these cultures.

Figure $1 A$ shows a schematic illustration to this approach. The 7 spots of dried aggrecan and laminin on glass coverslips created a concentration gradient at the spot's border, with an increase in concentration toward the spot's edge. This phenomenon is referred to in physics as the "coffee ring" effect. In this case, the effect is created by the aggrecan spot solution evaporating in the 

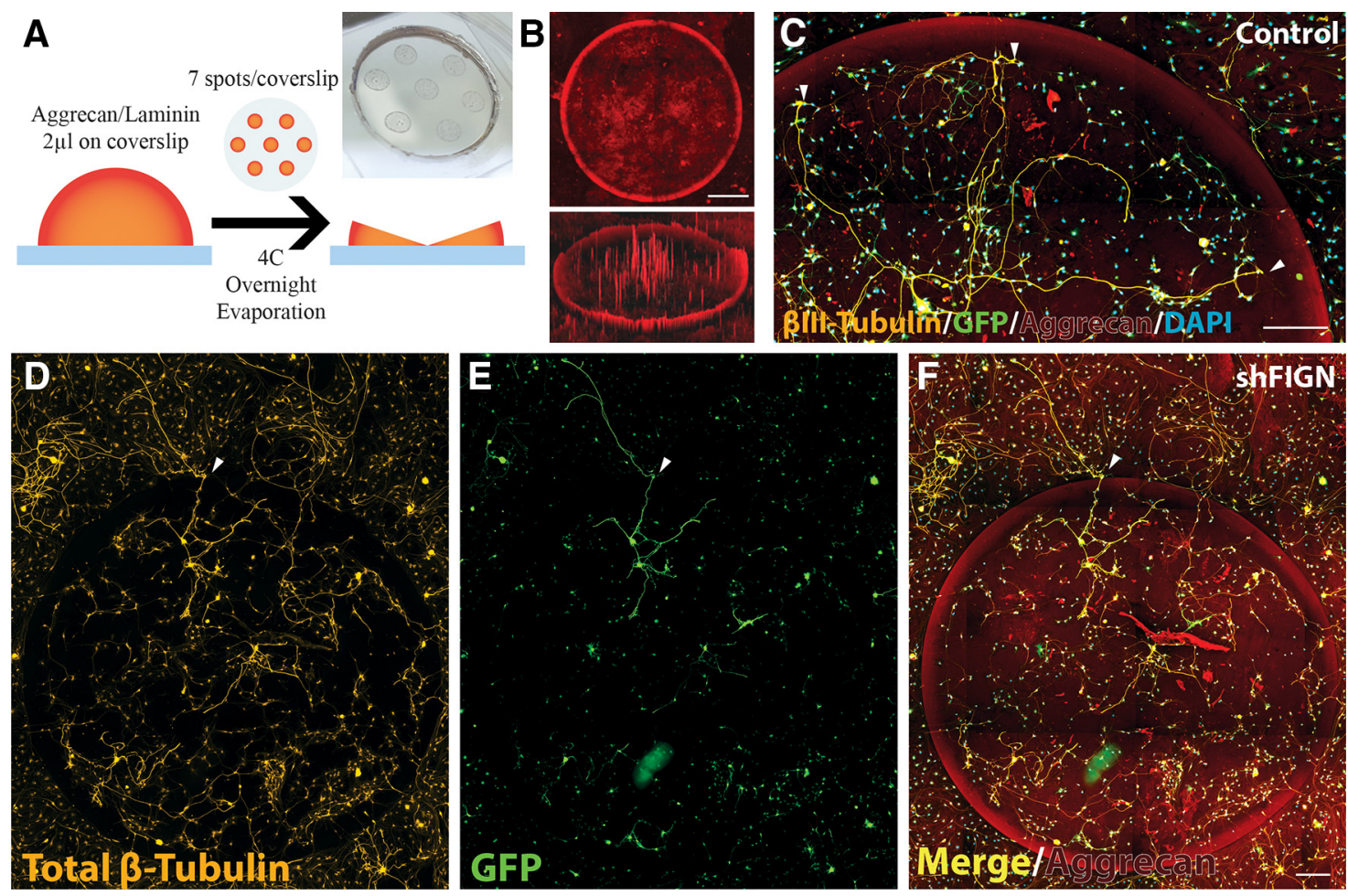

Figure 1. Aggrecan/laminin spot assay. $A$, The assay uses 7 spots of $2 \mu 10.7 \mathrm{mg} / \mathrm{ml}$ aggrecan and $5 \mu \mathrm{g} / \mathrm{ml} \mathrm{laminin} \mathrm{solution} \mathrm{pipetted} \mathrm{onto} \mathrm{the} \mathrm{surface} \mathrm{of} \mathrm{glass-bottomed} \mathrm{dishes.} \mathrm{The} \mathrm{spots} \mathrm{are} \mathrm{dried}$ overnight to produce a "coffee ring" effect that is visible to the eye. $\boldsymbol{B}$, The dry aggrecan spots have a high concentration of aggrecan at the edge of the spot and a gradually lower concentration moving toward the center, as can be visualized with fluorescence microscopy using an anti-aggrecan primary antibody and a Cy5 secondary antibody. The entire 20-mm-diameter insert of the glass-bottomed dishes was imaged. Shown here is a single spot with a diameter of $\sim 2000 \mu \mathrm{m}$ (top) and the same image using Carl Zeiss Zen 2.5D fluorescence intensity mapping, which reveals the "half-pipe" gradient of aggrecan at the spots edge. Scale bar, $500 \mu \mathrm{m}$. C, The aggrecan spots are inhibitory to axonal growth, as revealed by the $\beta$ III-tubulin/GFP double-labeled neuron at the center of the spot, extending growth cones outward. White arrows indicate the point at which the dystrophic growth cones stop as they approach the dense gradient of aggrecan. DAPI staining reveals there are other, non-neuronal cells on the aggrecan spot as well. Scale bar, $200 \mu \mathrm{m}$. D. With a general $\beta$-tubulin antibody, all of the cells that are on the aggrecan spots are stained, including satellite glia. $\boldsymbol{E}$, Neurons are strongly GFP ${ }^{+}$, whereas satellite glia and other non-neuronal cells are less so. $\boldsymbol{F}$, A merged image demonstrates information available, such as the number of neurons extending axons per spot, the number of satellite glia, and the small number of axons crossing (white arrowhead). Scale bar, $200 \mu \mathrm{m}$.

fume hood (as described in Materials and Methods, which could be visually observed on the surface of the glass coverslips; Fig. $1 A$ ). This was confirmed using immunofluorescence (Fig. $1 B$, top) and fluorescence-intensity heat mapping (Fig. 1B, bottom), which reveals an increasing concentration of aggrecan toward the outer rim. The uniformity of the aggrecan rim was consistent across dishes. Aggrecan staining may appear weaker in some images due to the loss of focus in the channel used to capture the aggrecan immunosignal because the tiling and autofocus features were set to prioritize the $\beta$-tubulin staining.

This pattern of aggrecan is similar to what an injured axon experiences; following an injury, the injured axon retracts from the site of injury and the axon's growth cone attempts regeneration into an increasingly inhibitory environment (Davies et al., 1997; Tom et al., 2004; Wu et al., 2016). Normally in this assay, the DRG axons of neurons both on and off the spot avoided the dense gradient of inhibitory substrate. Occasionally, axons attempted to enter this gradient; the growth cones of these axons became enlarged and dystrophic (Fig. 1C) as they approached the dense aggrecan border. Neurons and satellite glia were less likely to attach and grow on the spots, compared with off the spots (Fig. $1 D-F$ ). On very rare occasions, axons cross the spot's border (Fig. $1 D-F)$.

Satellite glia are more likely to cross (Fig. $1 D-F$ ). Where there was an increase in satellite glia on the aggrecan spot, antibody staining was reduced for aggrecan, thus revealing the ability of satellite glia to provide a suitable surface for axonal growth.
When axons did adhere to a spot and extend axons, they were often accompanied by satellite glia proximal to the cell body, similar to their in vivo environment. All cells avoided the dense gradient of aggrecan at the spot border. Neuron and satellite glia crossings were largely independent of one another. When axons crossed the dense aggrecan gradient, they were often devoid of a satellite glia association until crossing off the spot, where they would again associate with satellite glia. Immunofluorescence used quadruple labeling of aggrecan (CS-56), GFP, $\beta$-tubulin, and a DAPI nuclear counterstain (Fig. $1 F$ ).

\section{AAV5-based knockdown approach}

To investigate our hypothesis about fidgetin in a manner that could be translated to an in vivo model for spinal cord injury, we used AAV5-U6-shRNA-CMV-GFP, rather than the siRNA used in our previous studies. AAV5 is the adenovirus serotype previously shown to be effective at transducing adult DRGs in vitro and in vivo (Wu et al., 2017). Transduction efficiency of neurons in culture is high and the inclusion of GFP in the construct allowed visualization of the transduced neurons, with GFP diffusing along their axons at levels sufficient for tracing analysis (Fig. 2A$C)$. Unlike siRNA, the viral-driven shRNA expresses indefinitely and hence enables studies on longer-term cultures. The sequence of the shRNA was chosen from six different ones that were tried, based on knockdown efficiency of fidgetin cDNA in a screening method used by Vector Biolabs, whom we contracted for this work (see Materials and Methods; Fig. 2D). By $7 \mathrm{~d}$ after adding 

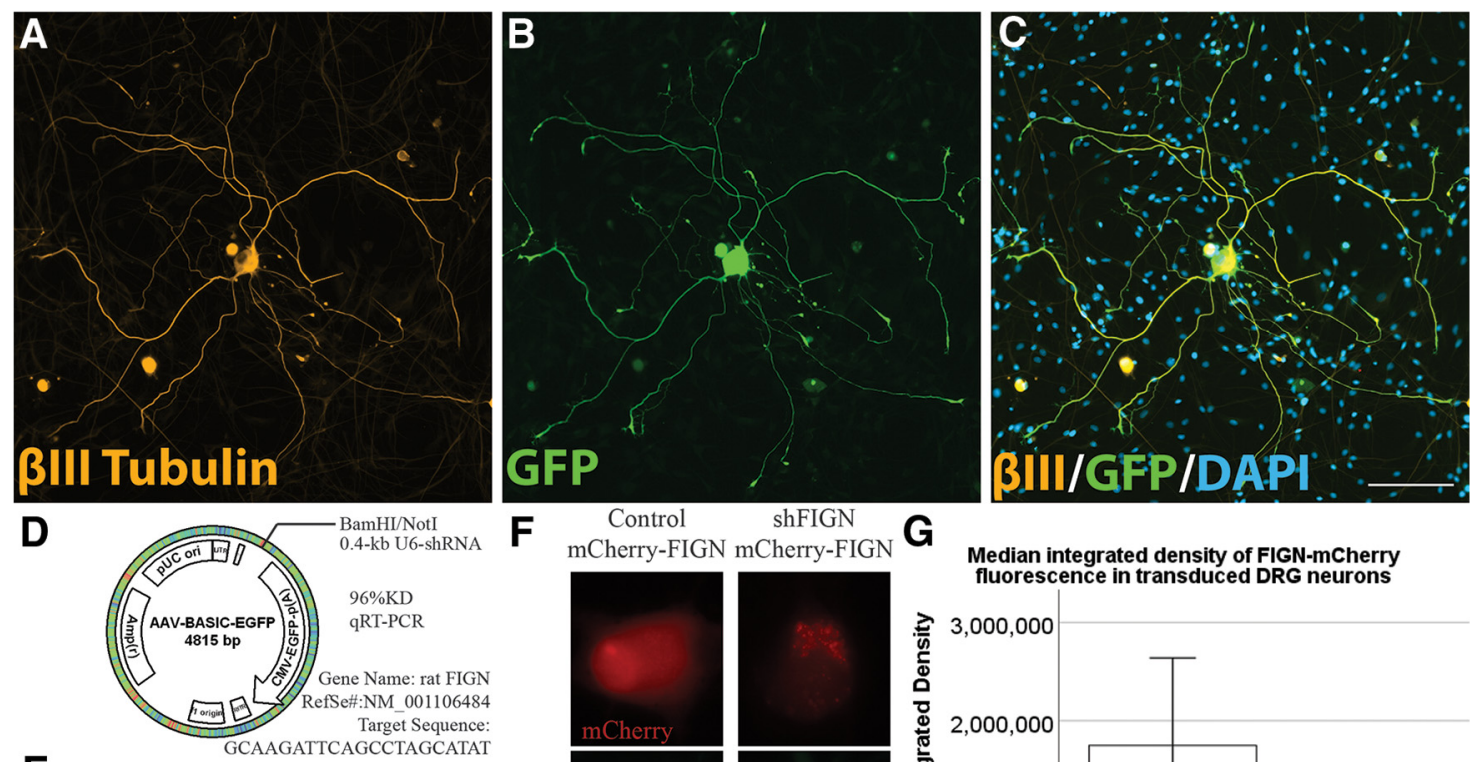

E

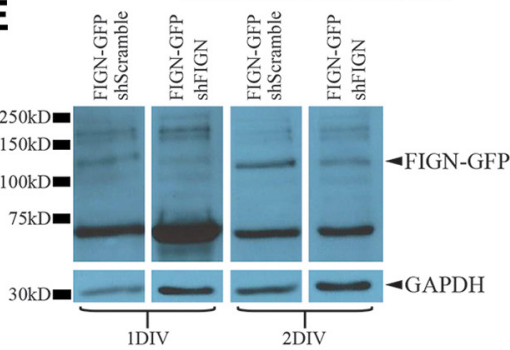

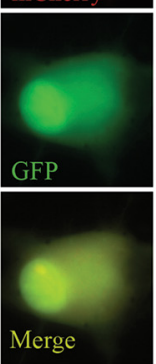

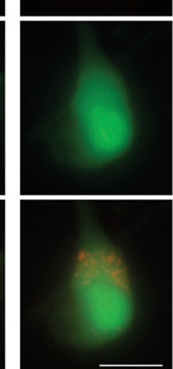

\section{G}

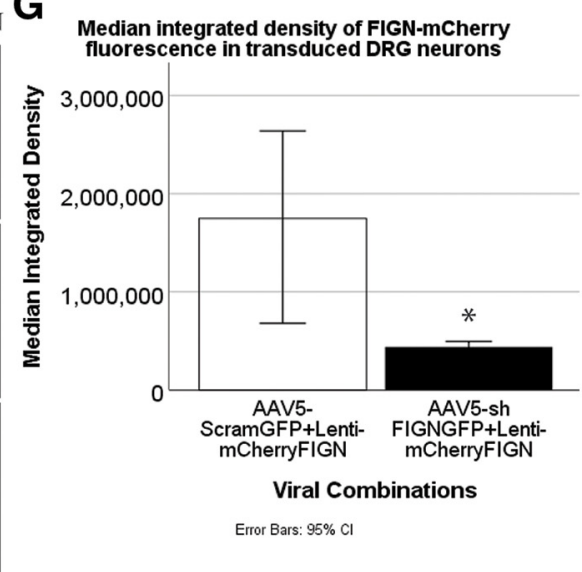

Median integrated density of FIGN-mCherry
fluorescence in transduced DRG neurons

Figure 2. AAV5-U6-shFIGN-CMV-GFP transduces neurons and knocks down fidgetin in vitro. Neurons were immunolabeled for the $(\boldsymbol{A})$ neuron-specific tubulin, $\boldsymbol{\beta}$ III-tubulin (yellow), $(\boldsymbol{B})$ the AAV5 reporter protein GFP, and (C) DAPI (to counterstain nuclei). These images demonstrate the high ectopic expression of GFP in transduced adult DRG neurons after 8 DIV. The primary cell cultures were heterogeneous, as seen when comparing $\beta$ III-tubulin immunolabeling with the DAPI staining, as there are multiple cells that are not $\beta$ III-tubulin ${ }^{+}$. All neurons were GFP ${ }^{+}$to some extent, but $<10 \%$ of the non-neuronal cells were detectably GFP ${ }^{+}$. Scale bar, $100 \mu \mathrm{m}$. D, The AAV-ready plasmid was used to construct our AAV5, and the rat fidgetin gene was targeted with a sequence that demonstrated $96 \%$ knockdown of rat fidgetin via qRT-PCR. E, RFL-6 cells were conucleofected with the AAV5-U6-shFIGN-CMV-GFP or AAV5-U6-controIRNA-CMV-GFP and plasmid containing fidgetin-GFP fusion protein. A commercially available fidgetin antibody detects ectopically expressed fidgetin-GFP at a molecular weight of $\sim 130 \mathrm{kDa}$. This band disappears after 24 or $48 \mathrm{~h}$ of ectopic coexpression of shRNA and fidgetin-GFP. F, Live-cell imaging was used to detect ectopically expressed mCherry (top panels, red) and GFP (middle panels, green) in adult DRG neurons, and a reduction in mCherry fluorescence was observed with cotransduction using shFIGN AAV5. Scale bar, $20 \mu \mathrm{m}$. G, mCherry-fidgetin was ectopically expressed via a lentivirus cotransduced with the shFIGN or control AAV5s in adult DRG primary cultures. Neurons were identified in live-cell imaging, and the mean integrated density of mCherry fluorescence was significantly reduced when cotransduced with the shFIGN AAV5: ${ }^{*} p<0.001$.

the virus to our adult rat DRG cultures, based on GFP fluorescence, $>80 \%$ of the total neurons strongly expressed the GFP reporter, with no statistical difference between the control and shFIGN viruses in expression efficiency or patterns. Fewer than $10 \%$ of total non-neuronal cells, such as satellite glia, expressed GFP at a level similar to neurons, indicating at least some differences among cell types in transduction efficiency or expression of the reporter.

Various commercially available antibodies as well as an inhouse antibody were tested for fidgetin detection. Endogenous fidgetin bands were not robust on Western blots with any available antibody, so we turned to ectopic expression of GFP-tagged fidgetin to confirm the capacity of the shRNA to knock down rat fidgetin from cells. One antibody was capable of reliably detecting ectopically expressed fidgetin-GFP in rat fibroblasts as well as the protein band's depletion when both the fidgetin-GFP and shFIGN vectors were conucleofected (with cell lysates collected at 24 and $48 \mathrm{~h}$ for Western blotting; Fig. 2E). To confirm the result in DRG neurons, we ectopically expressed fidgetinmCherry using a lentivirus cotransduced with our AAV5-U6shFIGN-CMV-GFP. A Mann-Whitney $U$ test was run to measure differences between AAV5 treatment (Control, $n=$ 50 cells; shFIGN, $n=50$ cells) and mCherry fluorescence using live cell imaging, thus measuring the efficiency of fidgetin shRNA to knock down ectopically expressed fidgetin in living adult DRG neurons after 3 DIV (Fig. $2 F$ ). Ectopically expressed mCherry median fluorescence integrated density was statistically significantly lower for shFIGN-AAV5:mCherryfidgetin lentivirus compared with Control-AAV5:lentivirusmCherry-fidgetin, $U=527, z=-4.984, p<0.001$ (Fig. $2 G$ ). These results demonstrate the ability of the shRNA to knock down fidgetin in living cells.

\section{Effects of fidgetin knockdown on satellite glia}

Because low levels of GFP expression were observed in satellite glial and other non-neuronal cells, we were able to quantify the total number of cells and the percentage of cellular area coverage per spot to assay for treatment effects, using epifluorescence microscopy. For the control group, cells avoided spots altogether (Fig. 3A1), populated spots sparsely (Fig. 3A2), or populated spots moderately (Fig. 3A3). When fidgetin was knocked down, cells populated the spots more densely (Fig. 3B4). The number of $\mathrm{DAPI}^{+}$cells was counted per aggrecan spot and averaged for the control $(n=107)$ and shFIGN $(n=106)$ treatment groups. The average number of DAPI ${ }^{+}$cells per spot was significantly greater for the shFIGN $(1070 \pm 49)$ group compared with the control 

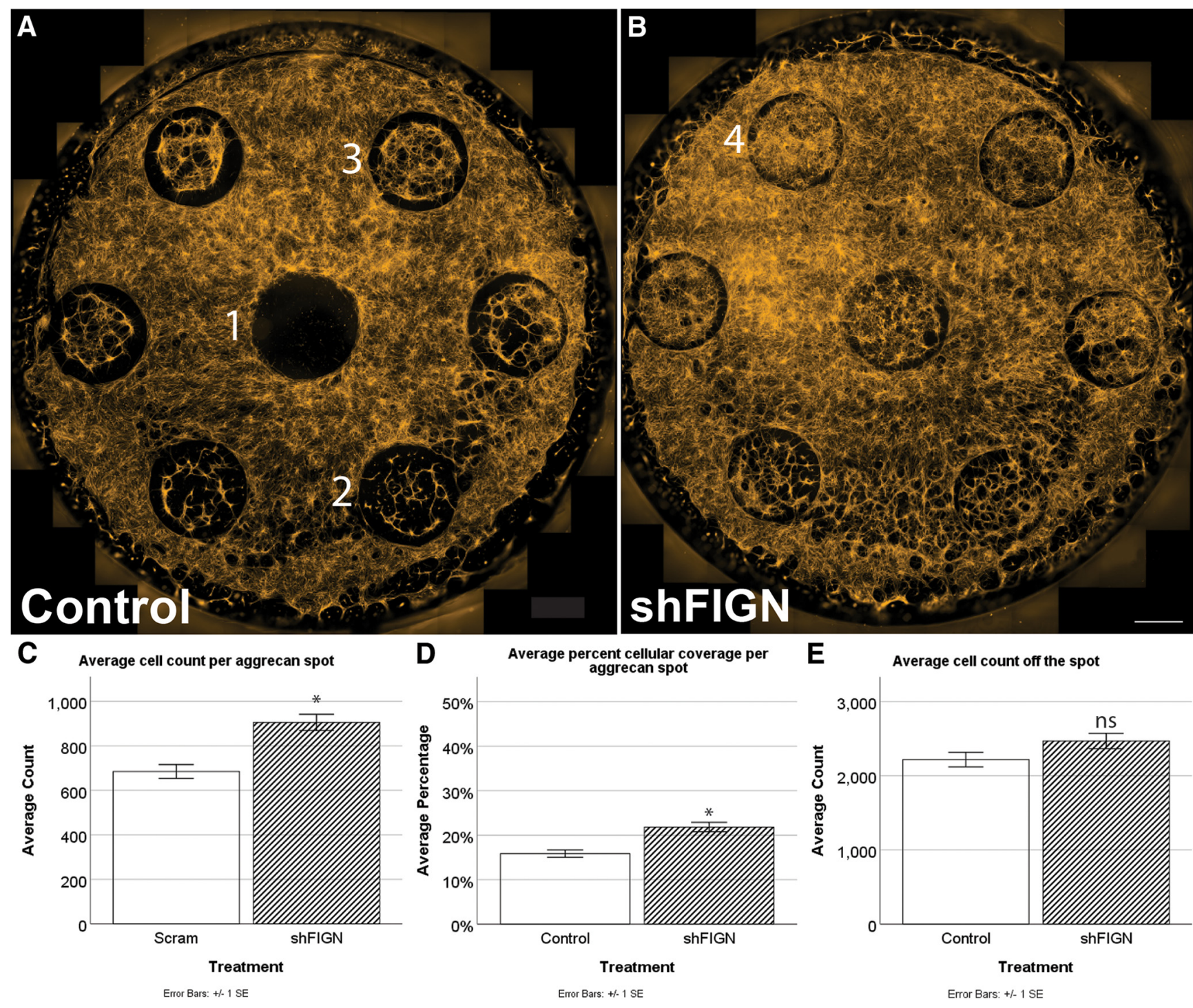

Figure 3. Satellite glia grow more avidly on aggrecan spots after fidgetin knockdown. $A, B$, Control and shFIGN-treated dishes differed in average cellular spot coverage. The aggrecan spots had minimal (1), sparse (2), or moderate (3) cellular coverage. Strong (5) cellular spot coverage was observed in the shFIGN AAV5 treatment dishes. Scale bar, $1000 \mu \mathrm{m}$. C, The number of DAPI ${ }^{+}$nuclei were counted per aggrecan spot, and the number of spots increased with fidgetin knockdown: ${ }^{*} p<0.001$. D, Total $\beta$-tubulin was immunolabeled, and Rols were drawn around each aggrecan spot to measure the average area covered with tubulin fluorescence. The shFIGN AAV 5 resulted in more aggrecan spot coverage of tubulin: ${ }^{*} p<0.001$. $E$, The same ROIs were shifted to areas off the spot, and there was no significant difference in cell counts between groups.

$(831 \pm 43)$ group, $t_{(211)}=-3.623, p<0.001$ (Fig. 3C). The average percentage area coverage per aggrecan spot was measured for control $(n=158)$ and shFIGN $(n=164)$ treatment group's spots. There was a significant increase in cellular spot coverage for the shFIGN (22 $\pm 1 \%$ ) group compared with control $(16 \pm 1 \%), t_{(320)}=-4.469, p<0.001$ (Fig. $\left.3 D\right)$. Average cell counts off-the-spot using the same ROIs are not significantly different between treatment groups (Fig. 3E).

Satellite glial cells engulf DRG neuronal cell bodies and axons to provide beneficial support (Hanani, 2010). Early in our studies, we decided not to include a mitotic inhibitor, such as cytosine arabinoside, in our cultures and not to use a neuron-specific promoter in our shRNA constructs because satellite glia are intimately associated with DRG neurons in vivo (Zhou and Rush, 1996; Zhou et al., 1999) and would be affected in future work by tools, such as drugs that are not neuron-specific. Satellite glia were normally not as hindered in crossing the spot's border as were the axons. Cellular processes extended by non-neuronal cells were morphologically similar to axons but did not exhibit dystrophic ends as they approached and crossed the aggrecan spot's border. Fidgetin knockdown increased the total number of cells on the spot (but not off the spot), presumably due to either greater proliferation or greater adherence of non-neuronal cells to the aggrecan. However, shFIGN did not enhance the crossing of satellite glia, as was the case with the axons. Control dishes had an average of $60( \pm 5)$ cells crossing per spot, whereas fidgetin knockdown resulted in an average of $74( \pm 6)$ crossings per spot; the difference was not significant $(p=0.74)$. Moreover, we observed that the majority of axon-crossing events did not have satellite glia associated with the axons (Fig. $1 D-F$ ). Thus, while we cannot dismiss the possibility that the positive effects on neurons are at least in part due to effects on non-neuronal cells, it would appear more likely that the effects on axonal growth and crossing events of the shFIGN are mainly due to fidgetin knockdown from the neurons themselves. 

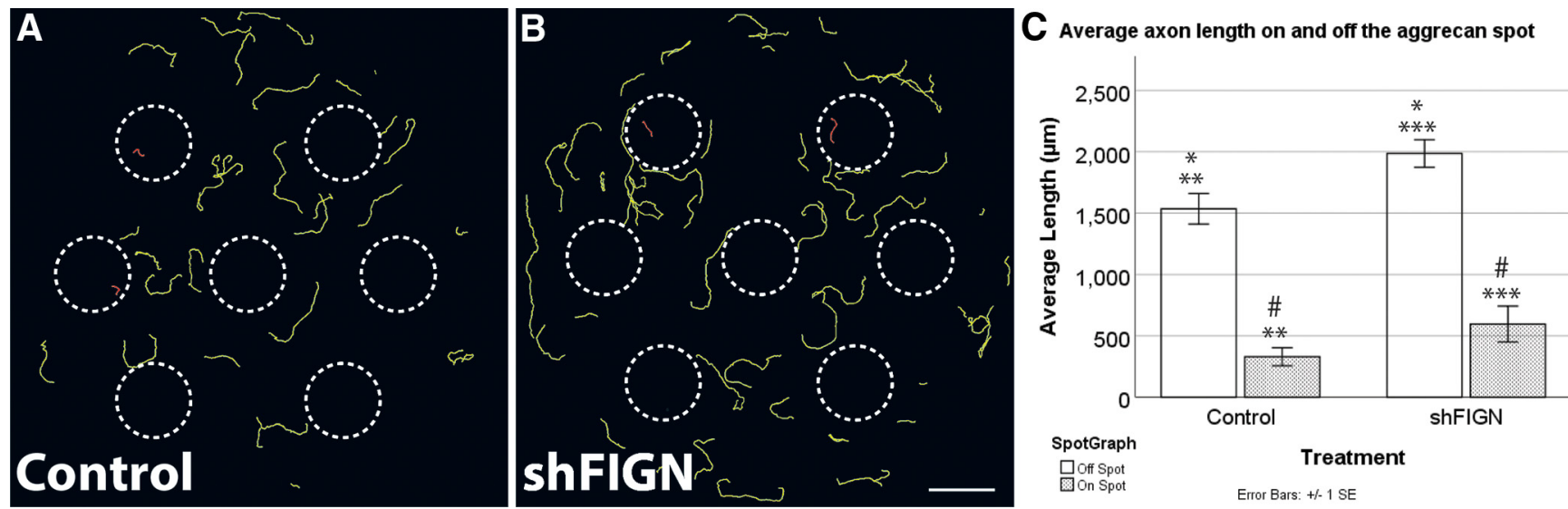

Figure 4. Fidgetin knockdown increases the average length of axons both on and off the aggrecan spots. $\boldsymbol{A}, \boldsymbol{B}$, Average lengths of control and shFIGN AAV5-treated axons were longer off-the-spot compared with on-the-spot, demonstrating the inhibitory effect of the aggrecan on axonal growth. After fidgetin knockdown, axons both on-the-spot and off-the-spot are obviously longer compared with control AAV5-treated axons. Dotted-line circles represent aggrecan spot tracings or ROls. Axons off-the-spot are traced in green and found outside the dotted-line circles. Axons on-the-spot are traced in red and found inside the dotted-line circles. Scale bar, $2000 \mu \mathrm{m}$ (the average diameter of the aggrecan spots). C, All groups had axons longer off-the-spot compared with on-the-spot: ${ }^{* *} p<0.001 ;{ }^{* *} p<0.001$. Axonal length off the aggrecan spot was significantly longer for the AAV5 shFIGN treatment group: ${ }^{*} p=0.005$. Fidgetin knockdown significantly increased axon length on the spots compared with the control group: ${ }^{\#} p<0.001$.

\section{Fidgetin knockdown promotes axonal crossing of the aggrecan border}

Aggrecan spots impaired axonal growth for both groups compared with axons off the spots (Fig. $4 A, B$ ). Average axonal length was measured in cultures immunolabeled for B-III tubulin (Fig. $4 C$ ). An independent-samples $t$ test was used to assess differences between the average axonal lengths on (Control, $n=57$; shFIGN, $n=77$ ) and off (Control, $n=244$; shFIGN, $n=343$ ) the aggrecan spots for each treatment group (Fig. 4A). The control group's axons were significantly longer off the spot $(1535 \pm 62 \mu \mathrm{m})$ compared with on the spot $(329 \pm 37 \mu \mathrm{m}), t_{(299)}=9.233, p<$ 0.001 . The shFIGN group's axons were significantly longer off the $\operatorname{spot}(1986 \pm 56 \mu \mathrm{m})$ compared with on the $\operatorname{spot}(596 \pm 73 \mu \mathrm{m})$, $t_{(418)}=11.247, p<0.001$.

Both on and off the spots, axons qualitatively appeared to be longer after fidgetin knockdown compared with the control group's axons (Fig. $4 A$ vs Fig. 4B). An independent-samples $t$ test was run to determine whether there were statistically significant differences in axon length between control and shFIGN treatment groups. Off the spot, average axon length was significantly longer for the shFIGN treatment group $(1986 \pm 56 \mu \mathrm{m})$ compared with the control group's axon length $(1535 \pm 62 \mu \mathrm{m})$ by $451 \mu \mathrm{m}, t_{(585)}=-5.301, p<0.001$. On the spot, there was also a significant increase in average axon length for shFIGN ( $595 \pm 73$ $\mu \mathrm{m})$ compared with control (329 $\pm 37 \mu \mathrm{m})$, but fidgetin knockdown did not restore the length of axons to their length off the spots. Therefore, fidgetin knockdown affects the growth of axons, even in the presence of aggrecan (Fig. $4 B, C$ ). These results are consistent with our previous observations that axons crossing from laminin to an aggrecan strip grew markedly faster on both substrates (Austin et al., 2017).

Axonal crossing of the increasingly inhibitory gradient of aggrecan at the spots border was rare, as seen previously (Wu et al., 2016). In our cultures, axons of the control group exhibited classic avoidance behavior of the inhibitory gradient at the spots border. Axons grew away from the border completely or approached it and turned back toward the center of the spot where aggrecan was less concentrated (Fig. $5 A$, arrows). However, when fidgetin was knocked down, not only did axons more often cross the spot border, they readily grew against the concentration gra- dient of aggrecan (Fig. 5B, arrowheads). To quantify the effects of fidgetin knockdown on such crossing, we first determined whether there was an increase in the number of neurons extending axons on the aggrecan spots after knockdown. The average number of neurons with axons $>10 \mu \mathrm{m}$ per spot was acquired for control $(n=89)$ and shFIGN $(n=90)$ treatment groups. The shFIGN group had significantly more axon-bearing neurons $(2.3 \pm 0.3)$ per spot compared with the control group $(1.1 \pm 0.1)$, $t_{(177)}=-3.449, p=0.001$ (Fig. 5C).

Having established that neurons were twice as likely to extend axons when fidgetin was knocked down, we next explored whether fidgetin knockdown enhances crossing of the aggrecan spot border. Aggrecan spots were analyzed for $\mathrm{GFP}^{+} / \mathrm{BIIItub}^{+}$ axon crossing for both control $(n=111)$ and shFIGN $(n=112)$ treatment groups. Data were not normally distributed with a strong positive skew due to the high incidence of zero crossing events on aggrecan spots. Therefore, we used the nonparametric Mann-Whitney $U$ test to assess differences in median axon crossing between both treatment groups. The distributions of the crossings were graphed and found to be similar. Median axon crossing was significantly increased with fidgetin knockdown (1.0) compared with controls ( 0$), U=7,566, z=3.167, p=0.002$ (Fig. 5D). In Figure 5E, we used a stacked column graph to demonstrate the total sums of crossing as well as the orientation of crossing for both treatment groups. Axons either crossed from off-the-spot to on-the-spot, experiencing an abrupt change in surface substrate, or from on-the-spot to off-the-spot, moving through an increasingly inhibitory gradient of aggrecan. In the control treatment condition, axons more frequently crossed from off-the-spot to on-the-spot $(n=11)$ compared with on-the-spot to off-the-spot $(n=8)$. Overall, fidgetin knockdown increased the total number of crossing events from off-the-spot to on-the-spot $(n=29)$ and on-the-spot to off-the-spot $(n=41)$.

Thus, fidgetin knockdown improved the ability of axons to grow into the aggrecan from a favorable substrate and especially increased the ability of axons to grow from within the aggrecan against the gradient to enter the favorable substrate. Without fidgetin knockdown, the former challenge is met better than the latter challenge, whereas after fidgetin knockdown, the latter 


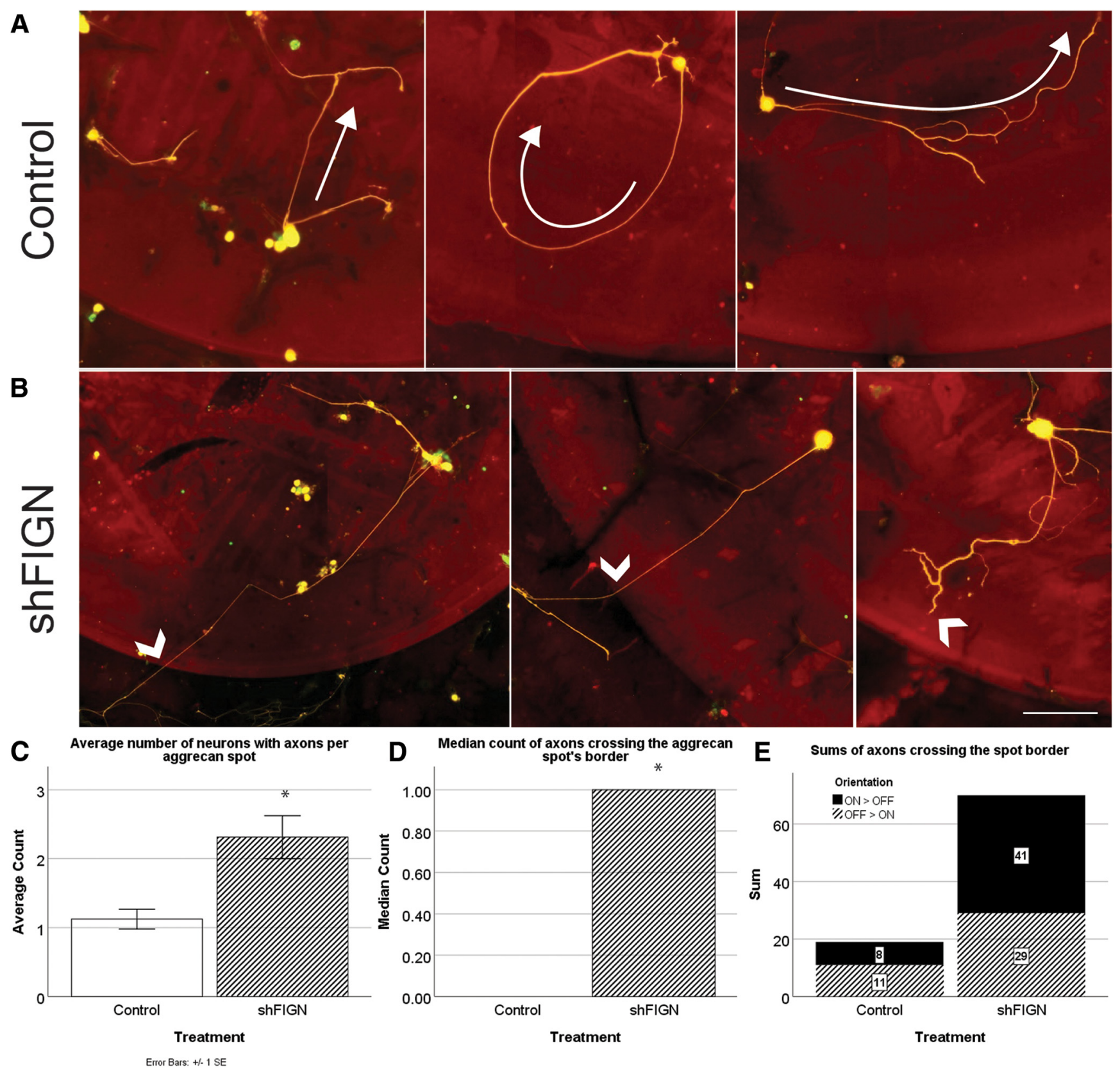

Figure 5. Fidgetin knockdown promotes neuronal growth on aggrecan spots and increases the number of axons crossing the gradient of aggrecan at the spot's border. $\boldsymbol{A}$, Axons transduced with control AAV5 avoided the border of the aggrecan spots. Many axons avoided the border altogether (left). Others grow toward and turn away from the aggrecan spot border (center). Neurons closer to the center of the circle project axons inward toward the lower concentration of aggrecan (right). B, Axons of neurons transduced with shFIGN AAV5 far more often grew through the spot's border (left and middle arrows). Some neurons readily extended bifurcated axons into the spot's border (right arrow). Scale bar, $100 \mu \mathrm{m}$. $C$, The average number of $\beta \mathrm{III}$-tubulin ${ }^{+} / \mathrm{GFP}^{+}$neurons with axons per aggrecan spot increased after fidgetin knockdown. $\boldsymbol{D}$, There was an increase in the average number of $\beta \mathrm{III}$-tubulin ${ }^{+} /$ $\mathrm{GFP}^{+}$axons crossing the inhibitory gradient of aggrecan at the spot's border: ${ }^{*} p<0.001$. $E$, The orientations of crossing axons were determined. Axons either cross from on-the-spot to off-the-spot (ON $>0 \mathrm{FF})$ or off-the-spot to on-the-spot (OFF $>0 N$ ). The total sums are represented in a stacked column graph, and there is a fivefold increase in crossing from on-the-spot to off-the-spot after fidgetin knockdown.

challenge is met better than the former challenge (although both are met better than without fidgetin knockdown). These results are encouraging as to the potential for fidgetin knockdown as a therapeutic treatment because axonal growth against the aggrecan concentration gradient is more reflective of the situation in vivo (Wu et al., 2016).

Effects of fidgetin knockdown on axonal regeneration in vivo The dorsal root crush assay is advantageous for our purposes, in part because it involves injured adult DRG axons attempting to regenerate into an inhibitory environment, and hence parallels the cell culture experiments described above. Furthermore, after completely crushing the dorsal roots spanning between C5 and T1, the animals display a "curled-arm" phenotype that results from total loss of sensation and can be used after surgery to confirm lesion completeness. Furthermore, spinal cord sections are cut in such a way that the dorsal roots are still attached, allowing for crushed roots to be observed for additional confirmation of a successful surgery. The crush completely interrupts the axons but does not completely sever the nerve, thus retaining a physical 

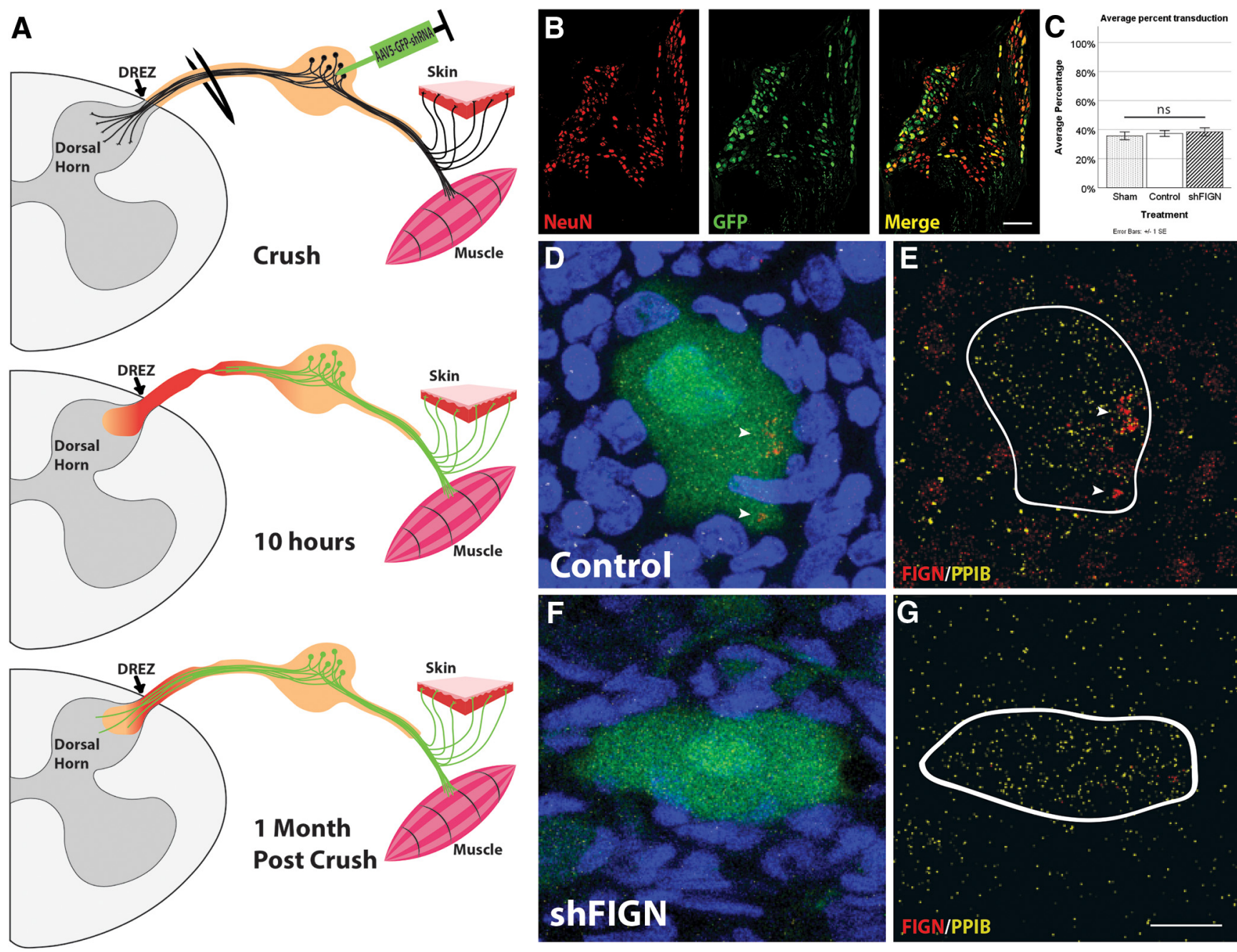

Figure 6. AAV5-U6-ShFIGN-CMV-GFP transduces neurons and knocks down fidgetin in vivo. A, A schematic of the in vivo dorsal root crush injury assay. Adult rats have their right dorsal roots crushed from $C 5$ to $\mathrm{T1}$, resulting in complete denervation of the front right paw as a result of the primary injury. The DRGs are injected with AAV5 using a glass micropipette. Hours later, neurons are transduced and the secondary injury begins. Animals are killed 1 month later, by which time DRG neurons are strongly expressing GFP, which is present in both their cell bodies and axons, allowing measurement of regeneration of GFP ${ }^{+}$axons beyond the DREZ and into the dorsal horn. $\boldsymbol{B}$, DRG neurons were identified using a NeuN-antibody, and transduced cells were identified using an antibody to GFP. The merged image exhibits strong GFP fluorescence mainly in neurons. C, Approximately $40 \%$ of the neurons were GFP ${ }^{+}$across all experimental groups with no significant (ns) differences. $\boldsymbol{D}, \mathrm{A} \mathrm{GFP}^{+}$DRG neuron is surrounded by DAPI ${ }^{+}$cells. $\boldsymbol{E}$, Using RNA Scope, the mRNA of fidgetin (red; white arrows) and PPIB (yellow; positive control) was revealed within the cytoplasm of neurons. Fidgetin mRNA was also located in the nucleus of surrounding non-neuronal cells. $\boldsymbol{F}$, Neurons injected with AAV5-shFIGN also express the GFP reporter. G, Fidgetin mRNA is knocked down from both the cytoplasm of neurons and nuclei of surrounding non-neuronal cells. Scale bar, $20 \mu \mathrm{m}$.

structure that supports axons to regenerate beyond the crush site. However, while axons are capable of modest regeneration across the crush site, they stop when they reach the DREZ and fail to reenter the spinal cord. This is thought to be due to both improper synapse formation and an injured tissue milieu filled with inhibitory molecules because of gliosis in both the peripheral nervous system and CNS induced by crushing the dorsal roots (Cheah et al., 2017). A more thorough description of surgery and animal care is provided in Materials and Methods, and a schematic detailing the in vivo assay can be found in Figure $6 A$.

The DRGs at C6-C8 were injected with virus, whereas C5 and T1 were not injected. This resulted in three injured and transduced ganglia flanked by two that were injured but nontransduced. The flanking two serve as immunohistological controls. No significant difference in tissue damage was found between crushed sites of sections included for analysis for both control and shFIGN AAV5 treatment groups; any injury sites that were severely injured were not included in the histological analysis. Control and shFIGN AAV5 were injected into the DRGs of adult rats and transduction efficiency assessed using anti-NeuN and anti-GFP antibodies (Fig. 6B). The NeuN labeling identified neurons, and the GFP antibody was used in conjunction with a Cy5 secondary so that both ectopically expressed GFP and immunolabeling of GFP could be used to confirm GFP expression. There was no statistical difference between the control $(36 \pm 3 \%)$ and shFIGN $(38 \pm 3 \%)$ AAV5 transduction efficiencies of DRG $\left(\mathrm{NeuN}^{+} / \mathrm{GFP}^{+}\right.$) neurons (Fig. 6C). In vivo, the AAV5 did not transduce satellite glial cells to an extent that resulted in robust GFP fluorescence, but the cell culture work suggests that at least some of these cells were nevertheless likely to have been transduced. $\mathrm{NeuN}^{+}$neurons were the predominant cells that showed strong, ectopically expressed, GFP fluorescence, and axons were clearly labeled. Together, Figure $6 B, C$ represents the $\mathrm{GFP}^{+}$neurons analyzed, as only $\mathrm{GFP}^{+}$axons were assessed for regeneration.

RNA Scope (ACDbio) was used to confirm fidgetin knockdown. This relatively new commercially available technology is based on RNA in situ hybridization with amplification steps that 

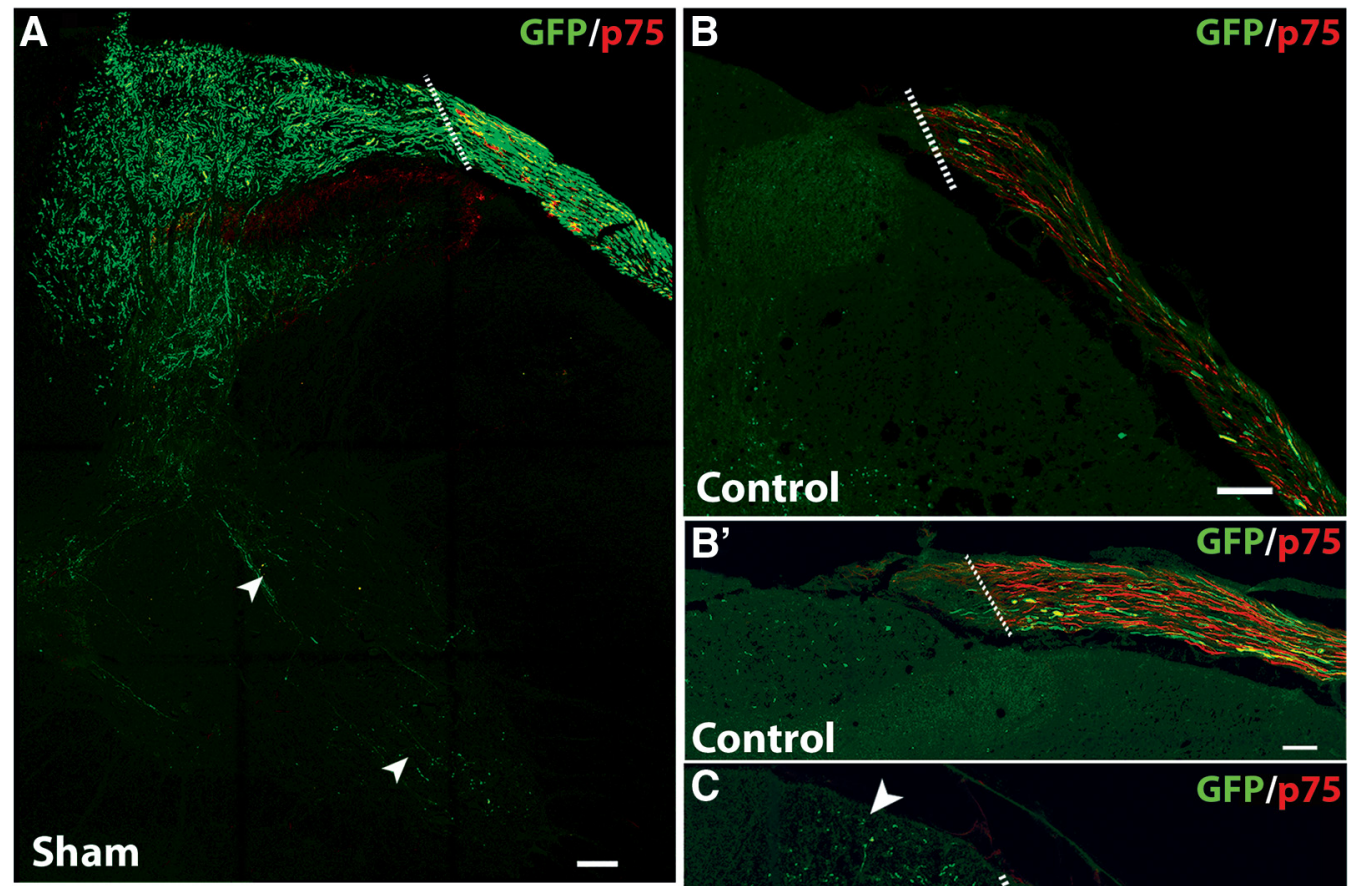

D Average number of GFP+ axons beyond the DREZ
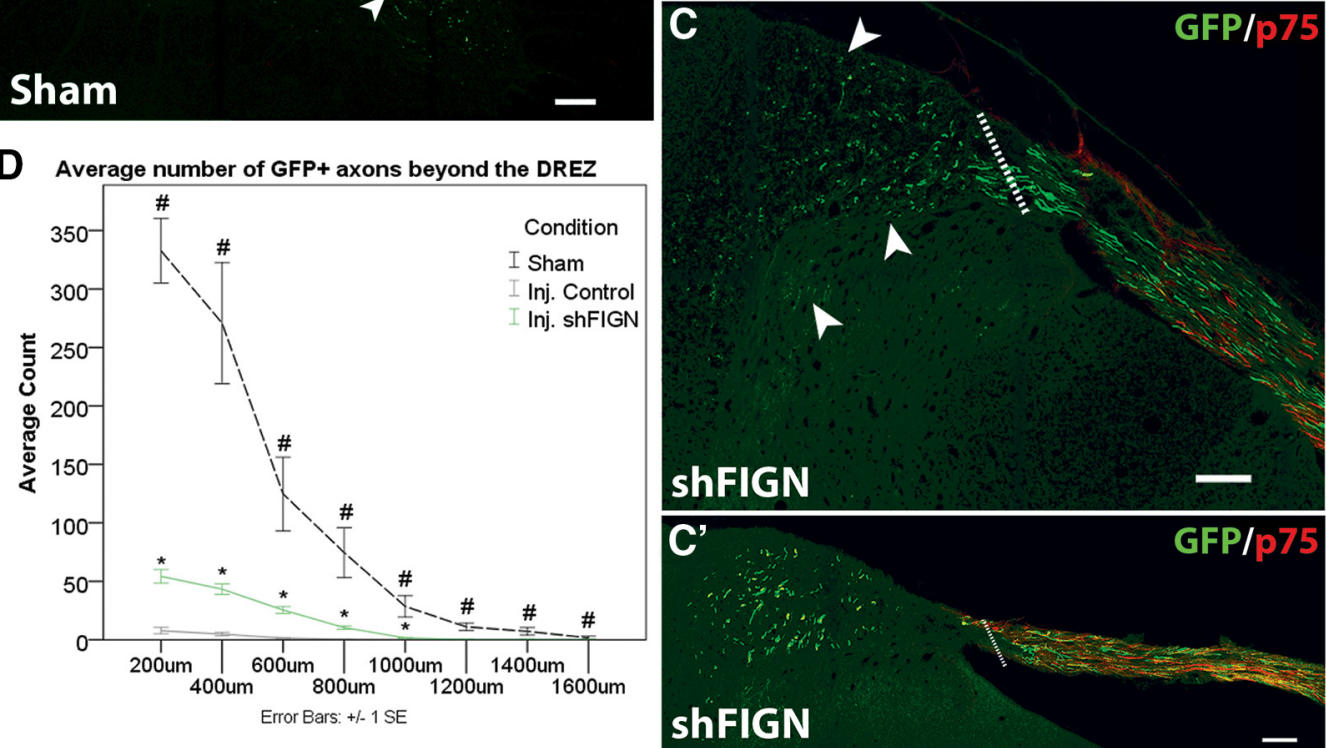

Figure 7. Fidgetin knockdown promotes axonal regeneration beyond the DREZ following dorsal root crush injury. Anti-GFP and anti-p75 antibodies were used to visualize GFP ${ }^{+}$axons and satellite glia, respectively. $\boldsymbol{A}$, An uninjured sham animal injected with AAV5 displays robust GFP expression in axons within the dorsal root that traverse the DREZ and extend deep into the dorsal horn, with some axons extending into the ventral horn of the spinal cord (white arrows). $\boldsymbol{B}, \boldsymbol{B}^{\prime}$, Following a dorsal root crush, axons rarely grow beyond the DREZ, demarcated by 775 immunolabeling. Dotted line indicates DREZ. C, $C^{\prime}$, After fidgetin knockdown, axons can be seen crossing the DREZ and regenerating into the dorsal horn (arrows). Scale bars, $100 \mu \mathrm{m}$. D. The average number of GFP ${ }^{+}$axons were quantified at $200 \mu \mathrm{m}$ intervals beyond the DREZ for an uninjured control animal ( $n=1$ animal, 6 tissue sections), control AAV5 animals ( $n=6$ animals, 44 tissue sections), and shFIGN AAV5 animals ( $n=6$ animals, 50 tissue sections). Sections with an intact dorsal root and GFP ${ }^{+}$axons were included in the analyses. Fidgetin knockdown significantly increased the amount of axonal regeneration beyond the DREZ and into the dorsal horn: ${ }^{*} p<0.05$; ${ }^{\#} p<0.05$.

allow for fluorescence labeling and detection of single mRNAs. We used a probe that targets the mRNA of fidgetin and a positive control (recommended by the manufacturer) that targets PPIB, which is a protein involved in protein folding widely expressed throughout many cell types. In Figure $6 D$, the $\mathrm{GFP}^{+}$neuron and DAPI $^{+}$nuclei are clearly observed, and Figure $6 E$ shows the dense labeling of fidgetin mRNA (red spots and clusters) within the neuronal cytoplasm, as well as fidgetin mRNA within the nucleus of neighboring non-neuronal cells. Both cells contain the positive control PPIB mRNA (yellow spots and clusters). Figure $6 F, G$ shows a tissue section from the treatment group with fidgetin knockdown, with virtually no fidgetin mRNA signal in either neuronal cytoplasm or non-neuronal nuclei. These results not only confirm that the shRNA knocks down fidgetin mRNA, but also indicate that the U6-promoter driven shRNA was expressed in most non-neuronal cells as well as neurons, despite their lack of detectable GFP. Therefore, the GFP-labeled axons only represent $40 \%$ of the neurons labeled by our virus with detectable levels of GFP, but not transduced, indicating that results observed in $\mathrm{GFP}^{+}$axons are likely occurring in unlabeled axons as well.

An animal that was subjected to the same surgical procedure as the injured animals, except for the dorsal root crushes, was used as a naive control to ascertain the extent of AAV5ectopically expressed $\mathrm{GFP}^{+}$axon labeling in the dorsal horn. The transduction efficiency $(43 \pm 3 \%)$ was not significantly different from the injured animal's transduction efficiency. There was extensive axon labeling in the sham animals, with some individual axons as far as $1600 \mu \mathrm{m}$ into the dorsal horn (Fig. $7 A, B$ ). Counts of $\mathrm{GFP}^{+}$axons decreased deeper in to the dorsal horn. In comparison, the injured control group rarely had any axons regener- 
ating beyond the DREZ, identified by Schwann cell labeling using anti-p75 antibody (Fig. 6C). Sections with an intact dorsal root and $\mathrm{GFP}^{+}$axons were included in the analysis. The data were approximately normally distributed, and homogeneity of variance was violated. Welch $t$ tests were performed to measure differences between the uninjured AAV5 animal $(n=1$ animal, 6 tissue sections) and both the injured control AAV5 ( $n=6$ animals, 44 tissue sections) and shFIGN AAV5 ( $n=6$ animals, 50 tissue sections) animals' average $\mathrm{GFP}^{+}$axon counts from 200 to $1000 \mu \mathrm{m}$ beyond the DREZ and into the dorsal horn. Both the injured control AAV5 and shFIGN AAV5 animals had significantly less axons in the dorsal horn compared with the uninjured sham; $200 \mu \mathrm{m}$ Uninjured Control (332.7 \pm 27.6$)$ versus Injured Control $(7.7 \pm 2.7), t_{(5.1)}=11.723, p<0.001 ; 200 \mu \mathrm{m}$ Uninjured Control $(332.7 \pm 27.6)$ versus Injured shFIGN $(54.2 \pm 5.9)$, $t_{(5.5)}=9.872, p<0.001 ; 400 \mu \mathrm{m}$ Uninjured Control (270.8 \pm $51.8)$ versus Injured Control $(4.8 \pm 1.5), t_{(5.0)}=5.134, p=0.004$; $400 \mu \mathrm{m}$ Uninjured Control $(270.8 \pm 51.8)$ versus Injured $\operatorname{shFIGN}(43.3 \pm 4.5), t_{(5.1)}=4.377, p=0.007 ; 600 \mu \mathrm{m}$ Uninjured Control $(124.7 \pm 31.4)$ versus Injured Control $(1.4 \pm 0.4)$, $t_{(5.0)}=3.920, p=0.011 ; 600 \mu \mathrm{m}$ Uninjured Control (124.7 \pm $31.4)$ versus Injured shFIGN $(25.4 \pm 3.0), t_{(5.1)}=3.144, p=$ 0.025; $800 \mu \mathrm{m}$ Uninjured Control $(74.5 \pm 21.4)$ versus Injured Control $(0.4 \pm 0.2), t_{(5.0)}=3.467, p=0.018 ; 800 \mu \mathrm{m}$ Uninjured Control $(74.5 \pm 21.4)$ versus Injured shFIGN $(10.4 \pm 1.4), t_{(5.0)}$ $=2.992, p=0.030 ; 1000 \mu \mathrm{m}$ Uninjured Control $(28.5 \pm 9.1)$ versus Injured Control $(0.0 \pm 0.0), t_{(5.0)}=3.115, p=0.026$; and $1000 \mu \mathrm{m}$ Uninjured Control $(28.5 \pm 9.1)$ versus Injured shFIGN $(1.6 \pm 0.4), t_{(5.0)}=2.940, p=0.032$. The uninjured control animal had axons that continued for another $600 \mu \mathrm{m} ; 1200 \mu \mathrm{m}$ Uninjured Control (11.0 \pm 3.1$) ; 1400 \mu \mathrm{m}$ Uninjured Control (7.1 \pm 3.2$)$; and $1600 \mu \mathrm{m}$ Uninjured Control (1.7 \pm 1.5$)$. The control and shFIGN AAV5 treatment groups extended a maximum distance of $1000 \mu \mathrm{m}$, and no statistics were necessary beyond this limit.

Fidgetin knockdown significantly increased the amount of axonal regeneration beyond the DREZ and into the dorsal horn compared with injured control axons (Fig. 7D); $200 \mu \mathrm{m}$ Injured Control $(7.7 \pm 2.7)$ versus Injured shFIGN $(54.2 \pm 5.9), t_{(92)}=$ $-6.889, p<0.001 ; 400 \mu \mathrm{m}$ Injured Control $(4.8 \pm 1.5)$ versus Injured shFIGN $(43.3 \pm 4.5), t_{(92)}=-7.198, p<0.001 ; 600 \mu \mathrm{m}$ Injured Control $(1.4 \pm 0.4)$ versus Injured shFIGN $(25.4 \pm 3.0)$, $t_{(92)}=-7.696, p<0.001 ; 800 \mu \mathrm{m}$ Injured Control $(0.4 \pm 0.2)$ versus Injured shFIGN $(10.4 \pm 1.4), t_{(92)}=-6.530, p<0.001$; $1000 \mu \mathrm{m}$ Injured Control $(0.1 \pm 0.1)$ versus Injured shFIGN $(1.6 \pm 0.4), t_{(92)}=-3.780, p<0.001$. At $200 \mu \mathrm{m}$, shFIGN had an average count of $54.2 \pm 6$ axons, and the control group had $7.7 \pm$ 2.7 , which is a sevenfold increase compared with injured control AAV5 animals (Fig. 7C,D). These data support our in vitro findings that fidgetin knockdown can promote axonal regeneration across a nonpermissive border and to extend beyond a site of injury. However, the knockdown alone, at least to the degree that our methods were able to achieve, did not result in obvious improvement in the animal voluntarily placing weight on its front right paw (data not shown), which we consider requisite in our workflow plan for moving to behavioral testing (see Discussion).

\section{Discussion}

Regeneration of injured nerves in the CNS is among the greatest limitations in the ability of the vertebrate body to repair itself, as well as one of the most perplexing unmet therapeutic challenges of contemporary biomedical research. Researchers have been testing approaches, such as disinhibition of growth-related signaling pathways, cell implants, nerve grafts, and degradation of inhibitory extracellular matrix to coax regeneration in the injured spinal cord, but these efforts have not yielded therapy for human patients (Massey et al., 2006; Park et al., 2008; Smith et al., 2009; Hanna et al., 2011; Nakamura and Okano, 2013). Augmenting growth-related signaling pathways involving genes, such as SOCS3, PTEN, and mTOR, can boost regeneration in experimental models, but these genes are also considered oncogenic with long-term studies lacking in assessment of potential negative side effects. With a focus to identify downstream targets that can circumvent such problems, the nerve regeneration field is gradually shifting toward cytoskeletal-based solutions. Our long-term interests align with recent enthusiasm in the regeneration field for microtubules, which can be thought of as a downstream bottleneck for axonal regeneration.

The most popular preclinical approach for altering the microtubule system to augment axonal regeneration has been to stabilize the microtubules (i.e., to dampen their dynamic properties). This can be done with anticancer drugs, such as taxol, which binds to the plus end of the microtubule and prevents subunit loss (Baas and Ahmad, 2013). Epothilones act similarly but are preferred because they cross the blood-brain barrier (Ruschel et al., 2015). In a plethora of studies published over the past several years, microtubule-stabilizing drugs have been shown to augment nerve regeneration in the culture dish as well as animal models, even leading to partial recovery of function in rats with injured spinal cords (Hellal et al., 2011; Sengottuvel et al., 2011; Ruschel et al., 2015; Li et al., 2017; Ruschel and Bradke, 2018; Sandner et al., 2018; Yang et al., 2018; Yin et al., 2018). However, an independent attempt failed to achieve notable regeneration or functional recovery, with only modest improvements apparently attributable to the effects of the drugs on non-neuronal cells contributing to the glial scar (Popovich et al., 2014). Other recent studies suggest that epothilones can actually be detrimental to functional recovery from spinal cord injury (Mao et al., 2017). To whatever degree that benefits may occur and through whatever mechanism, concern nevertheless exists about introducing microtubule-stabilizing drugs into the CNS, especially given the severe side effects of taxol treatment on peripheral nerves in patients taking the drug as cancer therapy (Scripture et al., 2006; Fukuda et al., 2017). Our laboratory has cautioned against abolishing the normal stable/labile domain structure of axonal microtubules with such drugs because each domain of the microtubule is functionally distinct and important (Baas and Ahmad, 2013). The counterargument to such concerns is that the drugs used at very low concentrations may promote the elongation of the labile domain of the microtubule without actually causing it to become as stable as the stable domain. However, even low levels of taxol have been shown to adversely affect microtubules in the distal region of the axon, where microtubules need to be especially labile (Gornstein and Schwarz, 2017). We have posited that a better approach is to tap into the growing body of knowledge of microtubule-related proteins to promote the elongation of the labile domain of the microtubule.

We have proposed that fidgetin inhibition might be a promising strategy because it leads to a significant increase in microtubule mass, which in turn leads to axonal growth (Leo et al., 2015; Austin et al., 2017). Because the newly added microtubule mass is labile, the microtubules not only become similar to those in juvenile axons, but the response magnifies the normal mechanisms by which axons mount a regenerative response (Kleele et al., 2014). The distal region of the axon and the growth cone may be especially relevant because microtubule engorgement of the 
growth cone is crucial for its consolidation into new axon (Lowery and Van Vactor, 2009). In addition, the capacity of the growth cone to turn properly in response to guidance cues is dependent upon the microtubules being labile (i.e., dynamic), with even very low doses of microtubule-stabilizing drugs impeding the ability of the growth cone to navigate properly (Williamson et al., 1996; Challacombe et al., 1997; Buck and Zheng, 2002). Thus, the hope is that fidgetin inhibition will not only enable the axon to grow better and to overcome inhibitory cues but might actually improve the capacity of the injured axon to find its appropriate target.

We have developed the regimen used in the present paper so that large bodies of data can be systematically gathered, with minimal experimenter bias, when various microtubule-related proteins are knocked down, inhibited, or overexpressed in neurons challenged to regenerate injured axons. The regimen starts with a medium-throughput cell culture assay that includes various challenges, such as axonal growth on a favorable substrate, axonal growth on an unfavorable substrate, growth of the axon from the favorable substrate to the unfavorable substrate, growth of the axon from the unfavorable substrate to the favorable substrate against a concentration gradient of the unfavorable substrate, and proliferation of non-neuronal cells, such as satellite glia on each substrate. The assay is amenable to modification, such as using CSPGs other than aggrecan, adding growth factors, such as NGF or BDNF, and using chondroitinase to degrade CSPGs. The large sample size allows our analyses to overcome a great deal of natural variation that arises from factors, such as multiple types of neurons and other cell types that comprise the DRG.

The AAV5 approach for altering gene expression can be directly translated from the cell culture assay to the next element of the regimen, which is an in vivo assay that also uses DRGs. The relatively short distance between the injected DRG cells bodies and injured axon in the dorsal root crush assay allows the axon of the DRG to be readily visualized with a GFP reporter, which may be too diluted to visualize in neurons with longer axons. In the future, we may consider adding other in vivo assays, such as those that require axonal regeneration over longer distances within the injured spinal cord, perhaps using a reporter with a stronger signal than GFP.

Fidgetin knockdown in vitro improved the capacity of the axon to overcome challenges designed to mimic those in the injured spinal cord. There was improvement in every parameter measured, and especially in the axon's capacity to grow against the aggrecan concentration gradient, which is the parameter most relevant to axonal regeneration in vivo (Wu et al., 2017). Our results suggest that these effects were mainly due to fidgetin reduction in the neurons, but we cannot entirely dismiss the possibility of a contribution from the non-neuronal cells, which were greater in number on the aggrecan spots when fidgetin was reduced. In vivo, fidgetin knockdown enabled regenerating axons to grow deep into the cord, overcoming their tendency to stall and form premature synapses upon entry (Di Maio et al., 2011). Indeed, the fidgetin-reduced axons displayed over 6 times as much axonal growth as their control counterparts. Even so, the animal showed no qualitative improvement in voluntary bearing of weight on its right paw; and hence, we did not pursue further behavioral analyses, nor did we investigate indicators of functional recovery, such as c-Fos expression, which is induced rapidly in postsynaptic neurons of the dorsal horn after electrical stimulation of DRG peripheral nerve fibers, thus indicating reinnervation (Wu et al., 2017).

With consensus in the spinal cord field shifting toward combinatorial approaches, it is not surprising that a single therapeutic intervention would be insufficient to produce functional recovery. In the future, we plan on characterizing the effects of knocking down fidgetin-related family members, such as fidgetin-like-2 (Charafeddine et al., 2015) or fidgetinlike-1 (Fassier et al., 2018), as well as other proteins whose depletion or inhibition would be expected to increase the axon's labile microtubule mass. These include certain kinesins that act as depolymerases to promote shortening of the microtubule from its plus end (Ghosh-Roy et al., 2012), as well as stathmin and SCG10, which promote disassembly of the microtubule by sequestering tubulin subunits (Grenningloh et al., 2004). We also plan to test molecular motor proteins kinesin-5 and kinesin-12, whose inhibition or knockdown is expected to assist in nerve regeneration by increasing microtubule mobility in the axon (Liu et al., 2010; Lin et al., 2011; Xu et al., 2015). Through these efforts, we envision developing a toolkit of microtubule-based therapies that can be mixed and matched (e.g., combining the best target to increase labile microtubule mass with the best target to increase microtubule mobility), and also used in combination with non-microtubulebased approaches, such as chondroitinase, grafts, exercise, or growth factors. It is our hypothesis that mechanistically defined tools with limited capacity to assist on their own will ultimately combine to provide a more robust and controllable clinical program of treatment for spinal cord injury than manipulation of broad upstream targets.

\section{References}

Austin TO, Matamoros AJ, Friedman JM, Friedman AJ, Nacharaju P, Yu W, Sharp DJ, Baas PW (2017) Nanoparticle delivery of fidgetin siRNA as a microtubule-based therapy to augment nerve regeneration. Sci Rep 7:9675.

Baas PW, Ahmad FJ (2013) Beyond taxol: microtubule-based treatment of disease and injury of the nervous system. Brain 136:2937-2951.

Baas PW, Black MM (1990) Individual microtubules in the axon consist of domains that differ in both composition and stability. J Cell Biol 111:495509.

Baas PW, Slaughter T, Brown A, Black MM (1991) Microtubule dynamics in axons and dendrites. J Neurosci Res 30:134-153.

Baas PW, Rao AN, Matamoros AJ, Leo L (2016) Stability properties of neuronal microtubules. Cytoskeleton (Hoboken) 73:442-460.

Bradke F, Fawcett JW, Spira ME (2012) Assembly of a new growth cone after axotomy: the precursor to axon regeneration. Nat Rev Neurosci 13:183193.

Buck KB, Zheng JQ (2002) Growth cone turning induced by direct local modification of microtubule dynamics. J Neurosci 22:9358-9367.

Challacombe JF, Snow DM, Letourneau PC (1997) Dynamic microtubule ends are required for growth cone turning to avoid an inhibitory guidance cue. J Neurosci 17:3085-3095.

Charafeddine RA, Makdisi J, Schairer D, O’Rourke BP, Diaz-Valencia JD, Chouake J, Kutner A, Krausz A, Adler B, Nacharaju P, Liang H, Mukherjee S, Friedman JM, Friedman A, Nosanchuk JD, Sharp DJ (2015) Fidgetin-like 2: a microtubule-based regulator of wound healing. J Invest Dermatol 135:2309-2318.

Cheah M, Fawcett JW, Andrews MR (2017) Dorsal root ganglion injection and dorsal root crush injury as a model for sensory axon regeneration. J Vis Exp 3:123.

Cho Y, Cavalli V (2012) HDAC5 is a novel injury-regulated tubulin deacetylase controlling axon regeneration. EMBO J 31:3063-3078.

Davies SJ, Fitch MT, Memberg SP, Hall AK, Raisman G, Silver J (1997) Regeneration of adult axons in white matter tracts of the central nervous system. Nature 390:680-683.

Delree P, Leprince P, Schoenen J, Moonen G (1989) Purification and culture of adult rat dorsal root ganglia neurons. J Neurosci Res 23:198-206.

Di Maio A, Skuba A, Himes BT, Bhagat SL, Hyun JK, Tessler A, Bishop D, Son YJ (2011) In vivo imaging of dorsal root regeneration: rapid immobilization and presynaptic differentiation at the CNS/PNS border. J Neurosci 31:4569-4582. 
Fassier C, Fréal A, Gasmi L, Delphin C, Ten Martin D, De Gois S, Tambalo M, Bosc C, Mailly P, Revenu C, Peris L, Bolte S, Schneider-Maunoury S, Houart C, Nothias F, Larcher JC, Andrieux A, Hazan J (2018) Motor axon navigation relies on fidgetin-like 1-driven microtubule plus end dynamics. J Cell Biol 217:1719-1738.

Fukuda Y, Li Y, Segal RA (2017) A mechanistic understanding of axon degeneration in chemotherapy-induced peripheral neuropathy. Front Neurosci 11:481.

Ghosh-Roy A, Goncharov A, Jin Y, Chisholm AD (2012) Kinesin-13 and tubulin posttranslational modifications regulate microtubule growth in axon regeneration. Dev Cell 23:716-728.

Gobrecht P, Andreadaki A, Diekmann H, Heskamp A, Leibinger M, Fischer D (2016) Promotion of functional nerve regeneration by inhibition of microtubule detyrosination. J Neurosci 36:3890-3902.

Gornstein EL, Schwarz TL (2017) Neurotoxic mechanisms of paclitaxel are local to the distal axon and independent of transport defects. Exp Neurol 288:153-166.

Grenningloh G, Soehrman S, Bondallaz P, Ruchti E, Cadas H (2004) Role of the microtubule destabilizing proteins SCG10 and stathmin in neuronal growth. J Neurobiol 58:60-69.

Gundersen RW, Barrett JN (1980) Characterization of the turning response of dorsal root neurites toward nerve growth factor. J Cell Biol 87:546-554.

Hanani M (2010) Satellite glial cells: more than just 'rings around the neuron.' Neuron Glia Biol 6:1-2.

Hanna AS, Côté MP, Houlé J, Dempsey R (2011) Nerve grafting for spinal cord injury in cats: are we close to translational research? Neurosurgery 68:N14-N15.

Hellal F, Hurtado A, Ruschel J, Flynn KC, Laskowski CJ, Umlauf M, Kapitein LC, Strikis D, Lemmon V, Bixby J, Hoogenraad CC, Bradke F (2011) Microtubule stabilization reduces scarring and causes axon regeneration after spinal cord injury. Science 331:928-931.

Kleele T, Marinković P, Williams PR, Stern S, Weigand EE, Engerer P, Naumann R, Hartmann J, Karl RM, Bradke F, Bishop D, Herms J, Konnerth A, Kerschensteiner M, Godinho L, Misgeld T (2014) An assay to image neuronal microtubule dynamics in mice. Nat Commun 5:4827.

Leo L, Yu W, D’Rozario M, Waddell EA, Marenda DR, Baird MA, Davidson MW, Zhou B, Wu B, Baker L, Sharp DJ, Baas PW (2015) Vertebrate fidgetin restrains axonal growth by severing labile domains of microtubules. Cell Rep 12:1723-1730.

Li Y, Tatsui CE, Rhines LD, North RY, Harrison DS, Cassidy RM, Johansson CA, Kosturakis AK, Edwards DD, Zhang H, Dougherty PM (2017) Dorsal root ganglion neurons become hyperexcitable and increase expression of voltage-gated T-type calcium channels (Cav3.2) in paclitaxel-induced peripheral neuropathy. Pain 158:417-429.

Lin S, Nazif K, Smith A, Baas PW, Smith GM (2015) Histone acetylation inhibitors promote axon growth in adult dorsal root ganglia neurons. J Neurosci Res 93:1215-1228.

Lin S, Liu M, Son YJ, Timothy Himes B, Snow DM, Yu W, Baas PW (2011) Inhibition of Kinesin-5, a microtubule-based motor protein, as a strategy for enhancing regeneration of adult axons. Traffic 12:269-286.

Liu M, Nadar VC, Kozielski F, Kozlowska M, Yu W, Baas PW (2010) Kinesin12 , a mitotic microtubule-associated motor protein, impacts axonal growth, navigation, and branching. J Neurosci 30:14896-14906.

Lowery LA, Van Vactor D (2009) The trip of the tip: understanding the growth cone machinery. Nat Rev Mol Cell Biol 10:332-343.

Mao L, Gao W, Chen S, Song Y, Song C, Zhou Z, Zhao H, Zhou K, Wang W, Zhu K, Liu C, Mei X (2017) Epothilone B impairs functional recovery after spinal cord injury by increasing secretion of macrophage colonystimulating factor. Cell Death Dis 8:e3162.

Massey JM, Hubscher CH, Wagoner MR, Decker JA, Amps J, Silver J, Onifer SM (2006) Chondroitinase ABC digestion of the perineuronal net promotes functional collateral sprouting in the cuneate nucleus after cervical spinal cord injury. J Neurosci 26:4406-4414.

Matamoros AJ, Baas PW (2016) Microtubules in health and degenerative disease of the nervous system. Brain Res Bull 126:217-225.

Nakamura M, Okano H (2013) Cell transplantation therapies for spinal cord injury focusing on induced pluripotent stem cells. Cell Res 23:70-80.

Park KK, Liu K, Hu Y, Smith PD, Wang C, Cai B, Xu B, Connolly L, Kramvis
I, Sahin M, He Z (2008) Promoting axon regeneration in the adult CNS by modulation of the PTEN/mTOR pathway. Science 322:963-966.

Podratz JL, Knight AM, Ta LE, Staff NP, Gass JM, Genelin K, Schlattau A, Lathroum L, Windebank AJ (2011) Cisplatin induced mitochondrial DNA damage in dorsal root ganglion neurons. Neurobiol Dis 41:661668.

Popovich PG, Tovar CA, Lemeshow S, Yin Q, Jakeman LB (2014) Independent evaluation of the anatomical and behavioral effects of taxol in rat models of spinal cord injury. Exp Neurol 261:97-108.

Ruschel J, Bradke F (2018) Systemic administration of epothilone D improves functional recovery of walking after rat spinal cord contusion injury. Exp Neurol 306:243-249.

Ruschel J, Hellal F, Flynn KC, Dupraz S, Elliott DA, Tedeschi A, Bates M, Sliwinski C, Brook G, Dobrindt K, Peitz M, Brüstle O, Norenberg MD, Blesch A, Weidner N, Bunge MB, Bixby JL, Bradke F (2015) Axonal regeneration. systemic administration of epothilone B promotes axon regeneration after spinal cord injury. Science 348:347-352.

Sandner B, Puttagunta R, Motsch M, Bradke F, Ruschel J, Blesch A, Weidner N (2018) Systemic epothilone D improves hindlimb function after spinal cord contusion injury in rats. Exp Neurol 306:250-259.

Scripture CD, Figg WD, Sparreboom A (2006) Peripheral neuropathy induced by paclitaxel: recent insights and future perspectives. Curr Neuropharmacol 4:165-172.

Sengottuvel V, Leibinger M, Pfreimer M, Andreadaki A, Fischer D (2011) Taxol facilitates axon regeneration in the mature CNS. J Neurosci 31:2688-2699.

Smith PD, Sun F, Park KK, Cai B, Wang C, Kuwako K, Martinez-Carrasco I, Connolly L, He Z (2009) SOCS3 deletion promotes optic nerve regeneration in vivo. Neuron 64:617-623.

Tom VJ, Steinmetz MP, Miller JH, Doller CM, Silver J (2004) Studies on the development and behavior of the dystrophic growth cone, the hallmark of regeneration failure, in an in vitro model of the glial scar and after spinal cord injury. J Neurosci 24:6531-6539.

Tran AP, Warren PM, Silver J (2018) The biology of regeneration failure and success after spinal cord injury. Physiol Rev 98:881-917.

Wang Z, Winsor K, Nienhaus C, Hess E, Blackmore MG (2017) Combined chondroitinase and KLF7 expression reduce net retraction of sensory and CST axons from sites of spinal injury. Neurobiol Dis 99:24-35.

Williamson T, Gordon-Weeks PR, Schachner M, Taylor J (1996) Microtubule reorganization is obligatory for growth cone turning. Proc Natl Acad Sci U S A 93:15221-15226.

Wu D, Klaw MC, Kholodilov N, Burke RE, Detloff MR, Côté MP, Tom VJ (2016) Expressing constitutively active rheb in adult dorsal root ganglion neurons enhances the integration of sensory axons that regenerate across a chondroitinase-treated dorsal root entry zone following dorsal root crush. Front Mol Neurosci 9:49.

Wu D, Klaw MC, Connors T, Kholodilov N, Burke RE, Côté MP, Tom VJ (2017) Combining constitutively active rheb expression and chondroitinase promotes functional axonal regeneration after cervical spinal cord injury. Mol Ther 25:2715-2726.

Xu C, Klaw MC, Lemay MA, Baas PW, Tom VJ (2015) Pharmacologically inhibiting kinesin-5 activity with monastrol promotes axonal regeneration following spinal cord injury. Exp Neurol 263:172-176.

Yang Y, Zhang X, Ge H, Liu W, Sun E, Ma Y, Zhao H, Li R, Chen W, Yuan J, Chen Q, Chen Y, Liu X, Zhang JH, Hu R, Fan X, Feng H (2018) Epothilone $\mathrm{B}$ benefits nigrostriatal pathway recovery by promoting microtubule stabilization after intracerebral hemorrhage. J Am Heart Assoc 7:e007626.

Yin W, Li X, Zhao Y, Tan J, Wu S, Cao Y, Li J, Zhu H, Liu W, Tang G, Meng L, Wang L, Zhu B, Wang G, Zhong M, Liu X, Xie D, Chen B, Ren C, Xiao Z, et al. (2018) Taxol-modified collagen scaffold implantation promotes functional recovery after long-distance spinal cord complete transection in canines. Biomater Sci 6:1099-1108.

Zhou XF, Rush RA (1996) Endogenous nerve growth factor is required for regulation of the low affinity neurotrophin receptor (p75) in sympathetic but not sensory ganglia. J Comp Neurol 372:37-48.

Zhou XF, Deng YS, Chie E, Xue Q, Zhong JH, McLachlan EM, Rush RA, Xian CJ (1999) Satellite-cell-derived nerve growth factor and neurotrophin-3 are involved in noradrenergic sprouting in the dorsal root ganglia following peripheral nerve injury in the rat. Eur J Neurosci 11:1711-1722. 